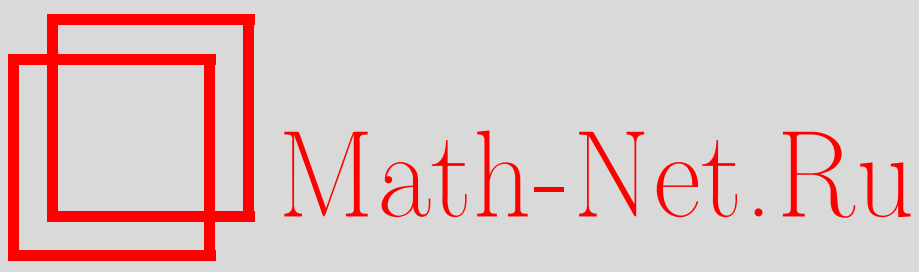

А. Л. Онищик, О. В. Платонова, Однородные супермногообразия, связанные с комплексным проективным пространством. I, Матем. сб., 1998, том 189, номер 2, $111-$ 136

DOI: https://doi.org/10.4213/sm295

Использование Общероссийского математического портала Math-Net.Ru подразумевает, что вы прочитали и согласны с пользовательским соглашением

http://www.mathnet.ru/rus/agreement

Параметры загрузки:

IP : 3.82 .47 .9

26 апреля 2023 г., 15:40:42 
УДК 515.177

\author{
А. Л. Онищик, О.В. Платонова
}

\title{
Однородные супермногообразия, связанные с комплексным проективным пространством. I
}

\begin{abstract}
Изучаются однородные и четно-однородные комплексные супермногообразия размерности $n \mid m$, редукцией которых является комплексное проективное пространство $\mathbb{C P}^{n}$. Предполагая, что $m \leqslant n$, классифицируются все расщепимые супермногообразия этого типа и вычисляем их 1-когомологии со значениями в касательном пучке, инвариантные относительно проективной группы.

Библиографиия: 15 названий.
\end{abstract}

\section{Введение}

Изучение однородных комплексных супермногообразий было начато в 80-х годах Маниным, который построил супермногообразия флагов, связанные с различньми сериями классических линейных суперагебр Ли (см. [1]). Имея в виду приложения к теоретической физике, Манин поставил и частично решил задачу классификации однородных комплексных супермногообразий вида $\left(\mathrm{Gr}_{4,2}, \mathscr{O}\right)$, где $\mathrm{Gr}_{4,2}$ - грассманово многообразие 2 -плоскостей в $\mathbb{C}^{4}$ или, иначе, модель Пенроуза (см. [1, гл. 5]). Представляется актуальной следующая задача: классифицировать все однородные комплексные супермногообразия вида $(M, \mathscr{O})$, где $M$ - заданное флаговое комплексное многообразие, т.е. однородное пространство вида $G / P$, где $G$-комплексная группа Ли, $P$ - ее параболическая подгруппа. В настоящей работе рассматривается случай, когда $M=\mathbb{C P}^{n}$ - комплексное проективное пространство.

Заметим, что известньм примером является супермногообразие П $\mathrm{Gr}_{n+1|n+1,1| 1}$ - П-симметричный суперграссманиан $1 \mid 1$-подпространств в $\mathbb{C}^{n+1 \mid n+1}$, определенньй в [1]. Отметим также, что ранее были получены следующие отдельные результаты: в [2] классифицированы все однородные супермногообразия вида $\left(\mathbb{C P}^{1}, \mathscr{O}\right)$ размерностей $1|1,1| 2,1 \mid 3$; в [3] построено однопараметрическое семейство нерасщепимых однородных супермногообразий вида $\left(\mathbb{C P}^{1}, \mathscr{O}\right)$ размерности $1 \mid 4$.

Наряду с однородньми, естественно рассматривать $\overline{0}$-однородные (или четно-однородные) супермногообразия, т.е. супермногообразия $(M, \mathscr{O})$, группа автоморфизмов которых транзитивно действует на их редукции $M$. Основной целью работы является классификация четно-однородных супермногообразий $(M, \mathscr{O})$ размерности $n \mid m$, где $M=\mathbb{C P}^{n}, n \geqslant 2$ и $n \geqslant m$, а также вычисление их групा когомологий со значениями в касательном пучке. В первой части мы предполагаем, что $(M, \mathscr{O})$ расщепимо; в этом случае задача сводится к теории линейных

Работа вьполнена при частичной поддержке Российского фонда фундаментальных исследований (грант № 95-01-01263). 
представлений. Мы проводим полную классификацию четно-однородных супермногообразий указанного типа и выделяем среди них однородные. Изучению наиболее интересного нерасшепимого случая будет посвящена вторая часть работы. Оно опирается на классификацию расщепимых однородных супермногообразий $(M, \mathscr{O})$ и на выгисление $G$-инвариантных 1-когомологий их касательного пучка, где $G=\mathrm{SL}_{n+1}(\mathbb{C})$ или $\mathrm{Sp}_{2 r}(\mathbb{C})$ (при $\left.n=2 r-1\right)$ - комплексная группа Ли, транзитивно действующая на $\mathbb{C P}^{n}$. Это вычисление также проводится в первой части работы в предположении, что $\mathrm{SL}_{n+1}(\mathbb{C})$ действует на $(M, \mathscr{O})$ в качестве группы автоморфизмов.

Результаты $\S 4$ и $\S 5$ анонсированы в [4], [5].

\section{§1. Предварительные сведения}

Термин “супермногообразие" мы употребляем в смысле Березина-Лейтеса, причем рассматривается комплексно-аналитический случай (см. [1]). Таким образом, супермногообразием размерности $n \mid m$ называется окольцованное пространство $(M, \mathscr{O})$, где $\mathscr{O}$ - пучок коммутативных комплексных супералгебр, локально изоморфное (с сохранением $\mathbb{Z}_{2}$-градуировок) окольцованному пространству вида $\left(U, \bigwedge_{\mathscr{F}_{n}}\left(\xi_{1}, \ldots, \xi_{m}\right)\right)$, где $U$ - открытое множество в $\mathbb{C}^{n}$ и $\mathscr{F}_{n}-$ пучок ростков голоморфных функций на $\mathbb{C}^{n}$. Пучок $\mathscr{O}$ называется структурнылм пучком, а числа $n$ и $m$ - соответственно, четной и нечетной размерностями супермногообразия $(M, \mathscr{O})$.

Супермногообразие $(M, \mathscr{O})$ называется расщепимым, если оно изоморфно супермногообразию вида $\left(M, \bigwedge_{\mathscr{F}} \mathscr{E}\right)$, где $\mathscr{E}-$ локально свободный аналитический пучок на комплексном многообразии $(M, \mathscr{F})$. Структурный пучок расщепимого супермногообразия обладает $\mathbb{Z}$-градуировкой

$$
\mathscr{O}=\bigoplus_{p=0}^{m} \mathscr{O}_{p}, \quad \mathscr{O}_{p}=\bigwedge_{\mathscr{F}}^{p} \mathscr{E}
$$

из которой заданная $\mathbb{Z}_{2}$-градуировка получается приведением по модулю 2 . В дальнейшем мы, как правило, будем опускать индекс $\mathscr{F}$ в обозначении внешних степеней и тензорных произведений аналитических пучков на $(M, \mathscr{F})$.

С любым супермногообразием $(M, \mathscr{O})$ можно связать некоторое расшепимое супермногообразие. Пусть $\mathscr{J} \subset \mathscr{O}$ - подпучок идеалов, порожденный подпучком $\mathscr{O}_{\overline{1}}$ нечетных элементов. Рассмотрим фильтрацию пучка $\mathscr{O}$

$$
\mathscr{O}=\mathscr{J}^{0} \supset \mathscr{J}^{1} \supset \mathscr{J}^{2} \supset \cdots
$$

степенями пучка $\mathscr{J}$ и присоединенный градуированньй пучок

$$
\operatorname{gr} \mathscr{O}=\bigoplus_{p \geqslant 0} \operatorname{gr}_{p} \mathscr{O}
$$

где

$$
\operatorname{gr}_{p} \mathscr{O}=\mathscr{J}^{p} / \mathscr{J}^{p+1}
$$

Оказывается, что $(M, \mathscr{F})$, где $\mathscr{F}=\operatorname{gr}_{0} \mathscr{O},-$ комплексное многообразие, а $\mathscr{E}=\operatorname{gr}_{1} \mathscr{O}$ - локально свободный аналитический пучок на нем. Более того, gr $\mathscr{O} \simeq \bigwedge_{\mathscr{F}} \mathscr{E}$, так 
что $(M, \operatorname{gr} \mathscr{O})$ - расщепимое супермногообразие. Будем называть его ретрактом супермногообразия $(M, \mathscr{O})$.

Обозначим через $\mathscr{T}=\mathcal{D}$ er $\mathscr{O}$ пучок дифференцирований структурного пучка $\mathscr{O}$. Пучок $\mathscr{T}$ является пучком супералгебр Ли относительно скобки Ли

$$
[u, v]=u v-(-1)^{p(u) p(v)} v u .
$$

Он называется касательным пучком, а его сечения - голоморфными векторными полями на $(M, \mathscr{O})$. Пространство голоморфных векторных полей $\Gamma(M, \mathscr{T})=$ $\mathfrak{v}(M, \mathscr{O})$ является супералгеброй Ли. Скобка Ли определяет также операцию $[\cdot, \cdot \cdot]$ в пространстве когомологий $H^{*}(M, \mathscr{T})=\bigoplus_{q \geqslant 0} H^{q}(M, \mathscr{T})$, которое становится градуированной алгеброй.

Если $(M, \mathscr{O})$ расшепимо, то $\mathscr{T}$ является $\mathbb{Z}$-градуированным пучком супералгебр Ли:

$$
\mathscr{T}=\bigoplus_{p \geqslant-1} \mathscr{T}_{p}
$$

где

$$
\mathscr{T}_{p}=\operatorname{Der}_{p} \mathscr{O}=\left\{\delta \in \mathscr{T}: \delta\left(\mathscr{O}_{q}\right) \subset \mathscr{O}_{q+p} \text { для всех } q \in \mathbb{Z}\right\} .
$$

Поэтому $\mathfrak{v}(M, \mathscr{O})=\bigoplus_{p \geqslant-1} \mathfrak{v}(M, \mathscr{O})_{p}$, где $\mathfrak{v}(M, \mathscr{O})_{p}=\Gamma\left(M, \mathscr{T}_{p}\right)$, есть $\mathbb{Z}$-градуированная супералгебра Ли. Градуировка возникает и в любой группе когомологий $H^{p}(M, \mathscr{T})$.

Вернемся к общему случаю и снабдим касательный пучок $\mathscr{T}$ супермногообразия $(M, \mathscr{O})$ фильтрацией:

$$
\mathscr{T}=\mathscr{T}_{(-1)} \supset \mathscr{T}_{(0)} \supset \cdots \supset \mathscr{T}_{(m)} \supset \mathscr{T}_{(m+1)}=0,
$$

где

$$
\mathscr{T}_{(p)}=\left\{\delta \in \mathscr{T}: \delta(\mathscr{O}) \subset \mathscr{J}^{p}, \delta(\mathscr{J}) \subset \mathscr{J}^{p+1}\right\} .
$$

Тогда $\left[\mathscr{T}_{(p)}, \mathscr{T}_{(q)}\right] \subset \mathscr{T}_{(p+q)}$, т.е. $\mathscr{T}$ - фильтрованный пучок супералгебр Ли.

Каждый $v \in \mathscr{T}_{(p)}$ переводит $\mathscr{J}^{q}$ в $\mathscr{J}^{q+p}$ и индуцирует при этом элемент пучка $\left(\mathscr{T}_{\mathrm{gr}}\right)_{p}$, где $\mathscr{T}_{\mathrm{gr}}=\mathcal{D} \operatorname{er}(\mathrm{gr} \mathscr{O})$. Таким образом, возникает гомоморфизм пучков $\sigma_{p}: \mathscr{T}_{(p)} \rightarrow\left(\mathscr{T}_{\mathrm{gr}}\right)_{p}$. Можно показать (см. [6]), что для любого $p \geqslant-1$ мы имеем точную последовательность

$$
0 \longrightarrow \mathscr{T}_{(p+1)} \longrightarrow \mathscr{T}_{(p)} \stackrel{\sigma_{p}}{\longrightarrow}\left(\mathscr{T}_{\mathrm{gr}}\right)_{p} \longrightarrow 0
$$

Отсюда следует, что градуированньй пучок супералгебр Ли $\mathscr{T}_{\mathrm{gr}}$ изоморфен градуированному пучку gr $\mathscr{T}$, присоединенному к фильтрации $(5)$. Если $(M, \mathscr{O})$ расщепимо, то фильтрация (5) пучка $\mathscr{T}$ совпадает с фильтрацией, ассоциированной с градуировкой (4), т.е.

$$
\mathscr{T}_{(p)}=\bigoplus_{r \geqslant p} \mathscr{T}_{r}
$$

Переходя к сечениям, мы видим, что супералгебра Ли векторных полей $\mathfrak{v}(M, \mathscr{O})$ допускает фильтрацию, связанную с фильтрацией (5) касательного пучка:

$$
\mathfrak{v}(M, \mathscr{O})=\mathfrak{v}(M, \mathscr{O})_{(-1)} \supset \mathfrak{v}(M, \mathscr{O})_{(0)} \supset \mathfrak{v}(M, \mathscr{O})_{(1)} \supset \cdots,
$$


где $\mathfrak{v}(M, \mathscr{O})_{(p)}=\Gamma\left(M, \mathscr{T}_{(p)}\right)$. Эта фильтрация определяет градуированную супералгебру Ли

$$
\widetilde{\mathfrak{v}}(M, \mathscr{O})=\bigoplus_{p \geqslant-1} \widetilde{\mathfrak{v}}(M, \mathscr{O})_{p}
$$

где $\widetilde{\mathfrak{v}}(M, \mathscr{O})_{p}=\mathfrak{v}(M, \mathscr{O})_{(p)} / \mathfrak{v}(M, \mathscr{O})_{(p+1)}$. Из (6) вытекает точная последовательность

$$
0 \longrightarrow \mathfrak{v}(M, \mathscr{O})_{(p+1)} \longrightarrow \mathfrak{v}(M, \mathscr{O})_{(p)} \stackrel{\sigma_{p}}{\longrightarrow} \mathfrak{v}\left(M, \mathscr{O}_{\mathrm{gr}}\right)_{p}, \quad p \geqslant-1
$$

приводящая к инъективному гомоморфизму градуированных супералгебр Ли $\widetilde{\sigma}: \widetilde{\mathfrak{v}}(M, \mathscr{O}) \rightarrow \mathfrak{v}\left(M, \mathscr{O}_{\mathrm{gr}}\right)$

Для расщепимых супермногообразий $(M, \bigwedge \mathscr{E})$ частичное описание пучка $\mathscr{T}_{p}$, $p \geqslant-1$, дает следующая точная последовательность локально свободных аналитических пучков на $M($ см. $[6])$ :

$$
0 \longrightarrow \mathscr{E}^{*} \otimes \bigwedge^{p+1} \mathscr{E} \stackrel{\alpha}{\longrightarrow} \mathscr{T}_{p} \stackrel{\beta}{\longrightarrow} \Theta \otimes \bigwedge^{p} \mathscr{E} \longrightarrow 0
$$

где $\Theta=\mathcal{D e r} \mathscr{F}$ - касательный пучок многообразия $M$, отображение $\beta$ сопоставляет каждому дифференцированию степени $p$ его ограничение на подпучок $\mathscr{F}$, a $\alpha$ отождествляет любой гомоморфизм пучков $\mathscr{E} \rightarrow \bigwedge^{p+1} \mathscr{E}$ с продолжающим его дифференцированием степени $p$, равным 0 на $\mathscr{F}$.

В частности, для $p=-1$ отображение $\alpha$ определяет изоморфизм

$$
\mathscr{T}_{-1} \simeq \mathscr{E}^{*}
$$

а в случае $p=0$ мы имеем точную последовательность

$$
0 \longrightarrow \mathscr{E}^{*} \otimes \mathscr{E} \stackrel{\alpha}{\longrightarrow} \mathscr{T}_{0} \stackrel{\beta}{\longrightarrow} \Theta \longrightarrow 0
$$

Пусть $\mathbf{E}$ - голоморфное векторное расслоение над $M$, соответствующее локально свободному пучку $\mathscr{E}$. Легко видеть, что $\mathscr{T}_{0}$ - пучок эндоморфизмов расслоения $\mathbf{E}$, а $\mathscr{E} * \otimes \mathscr{E}-$ его подпучок, состоящий из ростков эндоморфизмов, сохраняющих каждый слой. Первые члены точной последовательности когомологий, соответствующей (8), имеют вид

$$
0 \longrightarrow \mathfrak{g l}(\mathbf{E}) \stackrel{\alpha}{\longrightarrow} \mathfrak{v}(M, \mathscr{O})_{0} \stackrel{\beta}{\longrightarrow} \mathfrak{v}(M)
$$

где $\mathfrak{g l}(\mathbf{E})=\Gamma(M, \mathscr{E} * \otimes \mathscr{E})-$ алгебра Ли эндоморфизмов расслоения $\mathbf{E}$, сохраняющих слои, и $\mathfrak{v}(M)=\Gamma(M, \Theta)$ - алгебра Ли голоморфных векторных полей на $M$, причем $\alpha$ и $\beta$ - гомоморфизмы алгебр Ли. Если $M$ компактно, то имеем также соответствующую точную последовательность комплексных групп Ли

$$
e \longrightarrow \mathrm{GL}(\mathbf{E}) \longrightarrow \operatorname{Aut} \mathbf{E} \stackrel{\mu}{\longrightarrow} \operatorname{Bih} M
$$

где Aut $\mathbf{E}$ - группа автоморфизмов векторного расслоения $\mathbf{E}, \mathrm{GL}(\mathbf{E})$ - ее нормальная подгруппа, состоящая из автоморфизмов, сохраняющих слои (калибровочных преобразований), и $d \mu=\beta$ (см. [6]). Заметим, что GL(E) есть группа обратимых элементов ассоциативной алгебры $\mathrm{L}(\mathbf{E})$ эндоморфизмов расслоения $\mathbf{E}$. 
Отметим также, что алгебра $\mathfrak{g l}(\mathbf{E})$ (или $\mathrm{L}(\mathbf{E})$ ) всегда ненулевая (при условии $m>0)$. Действительно, она всегда содержит тождественный эндоморфизм id. Векторное поле $\varepsilon=\alpha(\mathrm{id})$ совпадает с градуируюшим дифференцированием пучка $\mathscr{O}$, действуюшим по формуле

$$
\varepsilon(f)=p f \text { для } f \in \mathscr{O}_{p} .
$$

Рассмотрим теперь важный для нас частный случай, когда $\mathbf{E}=\mathbf{T}(M)^{*}-$ кокасательное расслоение. В этом случае $\bigwedge \mathscr{E}=\Omega$-градуированньй пучок голоморфных форм на $M$. Описание пучка $\mathscr{T}=\mathcal{D} \operatorname{er} \Omega$ хорошо известно (см., например, [7]). В частности, точные последовательности (7) в этом случае расшепляются, т.е. сушествуют такие гомоморфизмы пучков векторных пространств $l: \Theta \otimes \Omega^{p} \rightarrow \mathscr{T}$, что $\beta l=\mathrm{id}$. Таким образом,

$$
\mathscr{T}_{p}=\alpha\left(\Theta \otimes \Omega^{p+1}\right) \oplus l\left(\Theta \otimes \Omega^{p}\right) \simeq \Theta \otimes \Omega^{p+1} \oplus \Theta \otimes \Omega^{p} .
$$

Пусть $(M, \mathscr{O})$ - некоторое супермногообразие, $x \in M$. Обозначим через $m_{x}$ максимальный идеал локальной супералгебры $\mathscr{O}_{x} \cdot \mathbb{Z}_{2}$-градуированное пространство $T_{x}(M, \mathscr{O})=\left(m_{x} / m_{x}^{2}\right)^{*}$ называется касательным пространством $\kappa(M, \mathscr{O})$ в точке $x$. Обозначая через $n_{x}$ максимальный идеал локальной алгебры $\mathscr{F} x$, где $\mathscr{F}=\mathscr{O} / \mathscr{J}$, мы имеем точную последовательность

$$
0 \longrightarrow \mathscr{J}_{x} \longrightarrow m_{x} \longrightarrow n_{x} \longrightarrow 0 .
$$

Из нее вытекает следующая точная последовательность:

$$
0 \longrightarrow \mathscr{J}_{x} / m_{x} \mathscr{J}_{x} \longrightarrow m_{x} / m_{x}^{2} \longrightarrow n_{x} / n_{x}^{2} \longrightarrow 0 .
$$

Векторное расслоение $\mathbf{E}$, соответствующее супермногообразию $(M, \mathscr{O})$, имеет в точке $x$ слой $\mathscr{J}_{x} / m_{x} \mathscr{J}_{x}=E_{x}$. Поскольку $T_{x}(M)=\left(n_{x} / n_{x}^{2}\right)^{*}$ есть касательное пространство к $M$ в точке $x$, получаем точную последовательность

$$
0 \longrightarrow T_{x}(M) \stackrel{i}{\longrightarrow} T_{x}(M, \mathscr{O}) \stackrel{p}{\longrightarrow} E_{x}^{*} \longrightarrow 0 .
$$

Очевидно, $i: T_{x}(M) \rightarrow T_{x}(M, \mathscr{O})_{\overline{0}}$ и $p: T_{x}(M, \mathscr{O})_{\overline{1}} \rightarrow E_{x}^{*}$-изоморфизмы векторных пространств.

Рассмотрим теперь вместо супермногообразия $(M, \mathscr{O})$ его ретракт $(M, \operatorname{gr} \mathscr{O})$. Максимальный идеал локальной супералгебры $(\operatorname{gr} \mathscr{O})_{x}=\mathscr{F}_{x} \oplus \bigoplus_{q \geqslant 1} \bigwedge^{q} \mathscr{E}_{x}$ имеет вид

$$
\widetilde{m}_{x}=n_{x} \oplus \bigoplus_{q \geqslant 1} \bigwedge^{q} \mathscr{E}_{x}
$$

причем

$$
\widetilde{m}_{x}^{2}=n_{x}^{2} \oplus n_{x} \mathscr{E}_{x} \oplus \bigoplus_{q \geqslant 2} \bigwedge^{q} \mathscr{E}_{x} .
$$

Отсюда $\widetilde{m}_{x} / \widetilde{m}_{x}^{2}=n_{x} / n_{x}^{2} \oplus E_{x}$, так что

$$
T_{x}(M, \operatorname{gr} \mathscr{O})=T_{x}(M) \oplus E_{x}^{*} .
$$


Очевидно, в этом случае $i$ и $p$ - это вложение $T_{x}(M) \rightarrow T_{x}(M, \operatorname{gr} \mathscr{O})$ и проекция $T_{x}(M, \operatorname{gr} \mathscr{O}) \rightarrow E_{x}^{*}$, соответственно.

Заметим, что любое дифференцирование $\delta \in T_{x}$ удовлетворяет условию $\delta\left(m_{x}^{2}\right) \subset m_{x}$ ипотому определяет линейное отображение $\widehat{\delta}: m_{x} / m_{x}^{2} \rightarrow \mathscr{O}_{x} / m_{x}=\mathbb{C}$. Это позволяет определить четное линейное отображение е $\mathrm{e}_{x}: \mathfrak{v}(M, \mathscr{O}) \rightarrow T_{x}(M, \mathscr{O})$ формулой

$$
\mathrm{ev}_{x}(v)=\widehat{v}_{x}
$$

причем $\widehat{v}_{x}$ называется значением векторного поля $v$ в точке $x$. Заметим, что (в противоположность классическому случаю) поле $v$, вообще говоря, не определяется однозначно своими значениями во всех точках $x \in M$.

Для любого голоморфного векторного расслоения $\mathbf{E} \rightarrow M$ будем обозначать тем же символом еv ${ }_{x}$ отображение пространства его голоморфных сечений $\Gamma(\mathbf{E})$ в слой $E_{x}, x \in M$, сопоставляюшее каждому сечению $s$ его значение $s(x) \in E_{x}$ в точке $x$.

Непосредственно проверяется следуюшее утверждение.

ПРЕДЛОЖЕНИЕ 1. Пусть $(M, \mathscr{O})$ - некоторое супермногообразие, $x \in M$. Тогда

(1) имеем

$$
\operatorname{ev}_{x}\left(\mathfrak{v}(M, \mathscr{O})_{(p)}\right)=0, \quad \text { если } \quad p \geqslant 1 ;
$$

(2) следующие диаграммы коммутативны:

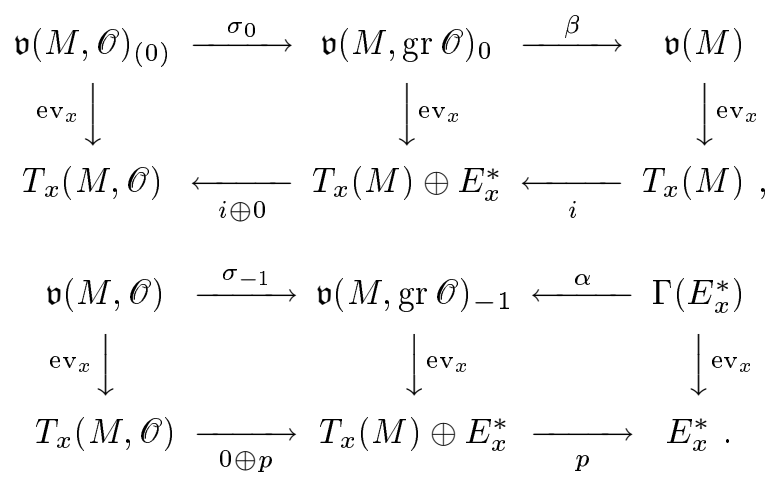

\section{§ 2. Транзитивные действия на супермногообразиях}

Пусть $M=(M, \mathscr{F})$ - связное комплексное многообразие. С каждьм голоморффньм действием $\Phi$ комплексной группы Ли $G$ на $M$ связано действие ее касательной алгебры Ли $\mathfrak{g}$, т.е. гомоморфизм $d \Phi: \mathfrak{g} \rightarrow \mathfrak{v}(M)$. Как известно из теории групп Ли, действие $\Phi$ транзитивно тогда и только тогда, когда действие $d \Phi$ транзитивно в том смысле, что отображение $\mathrm{ev}_{x}(d \Phi): \mathfrak{g} \rightarrow T_{x}(M)$ сюръективно для всех $x \in M$. Мы введем теперь аналогичным образом понятие транзитивного действия на супермногообразии, используя язык супералгебр Ли (см. [2], [3]).

Действием комплексной супералгебры Ли $\mathfrak{s}$ на комплексном супермногообразии $(M, \mathscr{O})$ называется любой гомоморфизм $\psi: \mathfrak{s} \rightarrow \mathfrak{v}(M, \mathscr{O})$. При рассмотрении действий мы обычно будем предполагать, что $M$ связно и компактно и что 
$\operatorname{dim} \mathfrak{s}<\infty$. Заметим, что из компактности многообразия $M$ вытекает, что $\operatorname{dim} \mathfrak{v}(M, \mathscr{O})<\infty$.

Пусть $(M, \mathscr{O})$ расщепимо. Действие $\psi$ супералгебры Ли s на $(M, \mathscr{O})$ назьвается градуированны.м, если $\mathfrak{s}-\mathbb{Z}$-градуированная супералгебра Ли и $\psi$ - гомоморфизм $\mathbb{Z}$-градуированных алгебр.

Если задано действие $\psi: \mathfrak{s} \rightarrow \mathfrak{v}(M, \mathscr{O})$, то с любым $x \in M$ связывается четное линейное отображение $\psi^{x}=\operatorname{ev}_{x} \psi: \mathfrak{s} \rightarrow T_{x}(M, \mathscr{O})$. Множество $\mathfrak{s}_{x}=\operatorname{Ker} \psi^{x}$ есть подалгебра в $\mathfrak{s}$, называемая стабилизатором точки $x$. Действие $\psi$ называется транзитивныц, если $\psi^{x}$ сюръективно для любого $x \in M$. В этом случае мы говорим, что $(M, \mathscr{O})$ - однородное пространство супералгебры Ли s. Действие $\psi$ называется $\overline{0}$-транзитивныц (или четно-транзитивныцм), если четная компонента $\psi_{\overline{0}}^{x}: \mathfrak{s}_{\overline{0}} \rightarrow T_{x}(M, \mathscr{O})_{\overline{0}}=T_{x}(M)$ отображения $\psi^{x}$ сюръективна для любого $x \in M$. Очевидно, транзитивное действие всегда $\overline{0}$-транзитивно.

Пусть $(M, \mathscr{O})$ - супермногообразие, причем $M$ компактно. Тогда имеется естественное действие $\psi=\mathrm{id}$ конечномерной супералгебры Ли $\mathfrak{v}(M, \mathscr{O})$ на $(M, \mathscr{O})$. Супермногообразие $(M, \mathscr{O})$ называется однородным (соответственно, $\overline{0}$-однородныцм или четно-однородным. ), если это действие транзитивно (соответственно, $\overline{0}$-транзитивно). Это значит, что отображение $\mathrm{ev}_{x}: \mathfrak{v}(M, \mathscr{O}) \rightarrow T_{x}(M, \mathscr{O})$ (соответственно, четная компонента этого отображения) сюръективно для любого $x \in M$.

Предположим, что задано действие $\psi$ супералгебры Ли $\mathfrak{s}$ на супермногообразии $(M, \mathscr{O})$. Мы хотим определить некоторое градуированное действие на расщепимом супермногообразии $(M, \mathrm{gr} \mathscr{O})$. Для этого заметим, что фильтрация (5) порождает фильтрацию

$$
\mathfrak{s}=\mathfrak{s}_{(-1)} \supset \mathfrak{s}_{(0)} \supset \cdots \supset \mathfrak{s}_{(m)} \supset \mathfrak{s}_{(m+1)}=0
$$

где

$$
\mathfrak{s}_{(p)}=\mathfrak{s} \cap \psi^{-1}\left(\Gamma\left(M, \mathscr{T}_{(p)}\right)\right) .
$$

Очевидно, $\mathfrak{s}$ становится фильтрованной супералгеброй Ли, а $\psi$ определяет гомоморфизм $\widetilde{\psi}$ соответствуюшей градуированной супералгебры Ли $\widetilde{\mathfrak{s}}$ в градуированную супералгебру Ли $\mathfrak{v}(M, \operatorname{gr} \mathscr{O})$, т.е. градуированное действие супералгебры Ли $\widetilde{\mathfrak{s}}$ на $(M, \operatorname{gr} \mathscr{O})$. При этом для любого $p \geqslant-1$ коммутативна следующая диаграмма:

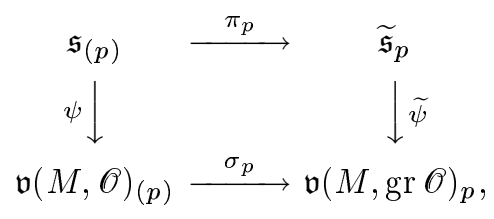

где $\pi_{p}: \mathfrak{s}_{(p)} \rightarrow \widetilde{\mathfrak{s}}_{p}-$ естественное отображение.

В частности, если рассматривать естественное действие $\psi=$ id супералгебры Ли $\mathfrak{s}=\mathfrak{v}(M, \mathscr{O})$ на компактном супермногообразии $(M, \mathscr{O})$, то $\mathfrak{s}_{(p)}=\mathfrak{v}(M, \mathscr{O})_{(p)}$, а градуированная супералгебра Ли $\widetilde{\mathfrak{s}}$ совпадает с супералгеброй $\widetilde{\mathfrak{v}}(M, \mathscr{O})$, определенной в $\S 1$. При этом $\widetilde{\psi}$ - это определенньй там же инъективный гомоморфизм $\widetilde{\sigma}: \widetilde{\mathfrak{v}}(M, \mathscr{O}) \rightarrow \mathfrak{v}(M, \operatorname{gr} \mathscr{O})$. В дальнейшем мы обычно будем отождествлять $\widetilde{\mathfrak{v}}(M, \mathscr{O})$ с образом этого гомоморфизма в $\mathfrak{v}(M, \mathrm{gr} \mathscr{O})$. 
ТЕОРема 1. Для любого действия $\psi$ супералгебры Ли $\mathfrak{s}$ на супермногообразии $(M, \mathscr{O})$ следующие условия әквивалентны:

(1) действие $\psi$ транзитивно;

(2) действие $\widetilde{\psi}$ супералгебры Ли $\widetilde{\mathfrak{s}}$ на супермногообразии $(M, \operatorname{gr} \mathscr{O})$ транзитивно;

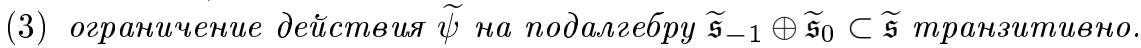

ДокаЗАТЕЛьство. Фиксируем $x \in M$. Из коммутативных диаграмм (12) и (15) следует, что

$$
\psi^{x}\left(\mathfrak{s}_{(0)}\right)=(i \oplus 0) \widetilde{\psi}^{x}\left(\widetilde{\mathfrak{s}}_{0}\right)
$$

Поскольку $\mathfrak{s}_{\overline{0}} \subset \mathfrak{s}_{(0)}$, мы видим, что $\psi_{\overline{0}}^{x}$ сюръективно тогда и только тогда, когда сюръективно $\widetilde{\psi}^{x}: \widetilde{\mathfrak{s}}_{0} \rightarrow T_{x}(M)$. Аналогично, используя (13) и (15), убеждаемся в том, что $\psi_{1}^{x}$ сюръективно тогда и только тогда, ког да сюръективно $\widetilde{\psi}^{x}: \widetilde{\mathfrak{s}}_{-1} \rightarrow E_{x}^{*}$. Отсюда видна эквивалентность утверждений (1) и (3). То, что (3) эквивалентно (2), следует из предложения 1 (1).

СлЕДСТВИЕ. Для любого компактного супермногообразия $(M, \mathscr{O})$ следующие условия әквивалентны:

(1) супермногообразие $(M, \mathscr{O})$ однородно;

(2) подалгебра $\widetilde{\mathfrak{v}}(M, \mathscr{O}) \subset \mathfrak{v}(M, \operatorname{gr} \mathscr{O})$ транзитивно действует на $(M, \operatorname{gr} \mathscr{O})$;

(3) подалгебра $\widetilde{\mathfrak{v}}(M, \mathscr{O})_{-1} \oplus \widetilde{\mathfrak{v}}(M, \mathscr{O})_{0} \subset \widetilde{\mathfrak{v}}(M, \mathscr{O})$ транзитивно действует на $(M, \operatorname{gr} \mathscr{O})$.

В частности, из однородности супермногообразия $(M, \mathscr{O})$ следует однородность его ретракта $(M, \operatorname{gr} \mathscr{O})$.

Очевидно, справедливы также аналогичные утверждения, касающиеся $\overline{0}$-транзитивных действий.

ТЕОРема 1'. Для любого действия $\psi$ супералгебры Ли э на супермногообразии $(M, \mathscr{O})$ следующие условия әквивалентны:

(1) действие $\psi$ является $\overline{0}-$ транзитивным;

(2) действие $\widetilde{\psi}$ является $\overline{0}-$ транзитивным;

(3) действие $\beta \widetilde{\psi}$ алгебрь Ли $\widetilde{\mathfrak{s}}_{0}$ на $M$ транзитивно.

СлЕДСТВИЕ. Для любого компактного супермногообразия $(M, \mathscr{O})$ следующие условия эквивалентны:

(1) супермногообразие $(M, \mathscr{O}) \overline{0}$-однородно;

(2) супералгебра Ли $\widetilde{\mathfrak{v}}(M, \mathscr{O})$ действует на $(M, \operatorname{gr} \mathscr{O}) \overline{0}-$ транзитивно;

(3) алгебра Ли $\widetilde{\mathfrak{v}}(M, \mathscr{O})_{0}$ действует на $M$ транзитивно.

В частности, из $\overline{0}$-однородности компактного супермногообразия $(M, \mathscr{O})$ следуют $\overline{0}$-однородность его ретракта $(M, \operatorname{gr} \mathscr{O})$ и однородность многообразия $M$.

\section{§3. Однородные векторные расслоения}

Пусть $\mathbf{E}$ - голоморфное векторное расслоение над связным компактным комплексным многообразием $M$. Тогда имеется естественный гомоморфизм комплекс- 
ных групп Ли $\mu:$ Aut $\mathbf{E} \rightarrow \operatorname{Bih} M$, входящий в (10). Расслоение $\mathbf{E}$ называется однородныцм, если действие $\mu$ группы Aut $\mathbf{E}$ на $M$ транзитивно. В этом случае $M-$ компактное комплексное однородное многообразие.

Предположим, что задан некоторый гомоморфизм $\Phi: G \rightarrow$ Aut $\mathbf{E}$ такой, что действие $\mu \Phi$ группы $G$ на $M$ транзитивно. Тогда $\mathbf{E}$ однородно. Будем говорить, что $\mathbf{E}$ однородно относительно $\Phi$ (или относительно $G$ ). Пусть $о \in M$ и $P=G_{o}$ - стабилизатор точки $o$ в $G$. Группа $P$ естественно действует в слое $E=E_{o}$, т.е. возникает голоморфное линейное представление $\varphi: P \rightarrow \mathrm{GL}(E)$. Известно, что расслоение $\mathbf{E}$ полностью определяется группой $G$, ее подгруппой $P$ и представлением $\varphi$, причем эти три объекта могут выбираться произвольно. При этом имеются естественные отождествления $M=G / P$ и $\mathbf{E}=\mathbf{E}_{\varphi}=G \times_{\varphi} E=(G \times E) / P$, где $P$ действует на $G \times E$ по формуле

$$
p(g, \xi)=\left(g p^{-1}, \varphi(p) \xi\right), \quad g \in G, \quad p \in P, \quad \xi \in E .
$$

Проекция расслоения $p: \mathbf{E}_{\varphi} \rightarrow M=G / P$ определяется формулой

$$
p(\overline{g, \xi})=g P
$$

где $(\overline{g, \xi}) \in \mathbf{E}_{\varphi}-P$-орбита пары $(g, \xi)$. Группа $G$ действует на $\mathbf{E}_{\varphi}$ по формуле

$$
h(\overline{g, \xi})=(\overline{h g, \xi}), \quad g, h \in G, \quad \xi \in E .
$$

Отметим также, что стандартным операциям над представлениями отвечают аналогичные операции над расслоениями с одной и той же базой. В частности,

$$
\begin{gathered}
\mathbf{E}_{\varphi^{*}}=\mathbf{E}_{\varphi}^{*}, \quad \mathbf{E}_{\varphi_{1}+\varphi_{2}}=\mathbf{E}_{\varphi_{1}} \oplus \mathbf{E}_{\varphi_{2}} \\
\mathbf{E}_{\varphi_{1} \varphi_{2}}=\mathbf{E}_{\varphi_{1}} \otimes \mathbf{E}_{\varphi_{2}}, \quad \mathbf{E}_{\bigwedge^{q} \varphi}=\bigwedge^{q} \mathbf{E}_{\varphi} .
\end{gathered}
$$

Пространство голоморфных сечений $\Gamma(\mathbf{E}) G$-однородного векторного расслоения обладает естественной структурой $G$-модуля. Она задается формулой

$$
(g s)(x)=\Phi(g)\left(s\left(g^{-1} x\right)\right), \quad g \in G, \quad s \in \Gamma(\mathbf{E}), \quad x \in M .
$$

Таким образом, по определению

$$
\mathrm{ev}_{x}(g s)=\Phi(g)\left(\mathrm{ev}_{g^{-1} x}(s)\right) .
$$

В частности, отображение $\mathrm{ev}_{o}: \Gamma(\mathbf{E}) \rightarrow E_{o}$ является $P$-эквивариантным.

Будем говорить, что расслоение $\mathbf{E}$ порождается подпространством $V \subset \Gamma(\mathbf{E})$, если отображение $\mathrm{ev}_{x}: V \rightarrow E_{x}$ сюръективно для любого $x \in M$. В случае, когда $V=\Gamma(\mathbf{E})$, мы говорим, что расслоение $\mathbf{E}$ порождается сечениями.

Теорема 2. Пусть $\psi$ - градуированное действие супералгебры Ли э на расщепимом супермногообразии $(M, \mathscr{O})$, где пучок $\mathscr{O}=\bigwedge \mathscr{E}$ определяется голоморфницм векторныц расслоением $\mathbf{E} \rightarrow M$. Тогда

(1) действие $\psi \overline{0}$-транзитивно тогда и только тогда, когда $\mathbf{E}$ однородно относительно односвязной группь Ли $S_{0}$ с касательной алгеброй $\mathfrak{s}_{0}$;

(2) $\overline{0}$-транзитивное действие $\psi$ транзитивно тогда и только тогда, когда $\mathbf{E}^{*}$ порождается подпространством $V=\alpha^{-1}\left(\psi\left(\mathfrak{s}_{-1}\right)\right) \subset \Gamma\left(\mathbf{E}^{*}\right)$.

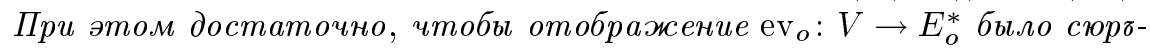
ективным для одной фиксированной точки о $\in$. 
ДокАЗАТЕЛЬСТво. Пусть $\Psi: S_{0} \rightarrow$ Aut $\mathbf{E}$ - такой гомоморфизм групп Ли, что $d \Psi=\psi$. Тогда имеем последовательность гомоморфизмов

$$
S_{0} \stackrel{\Psi}{\longrightarrow} \text { Aut } \mathbf{E} \stackrel{\mu}{\longrightarrow} \operatorname{Bih} M
$$

Поскольку $d(\mu \Psi)=\beta \psi$, утверждение (1) следует из теоремы $1^{\prime}$.

Из коммутативности диаграммы (13) следует, что $\mathrm{ev}_{x}(V)=p\left(\psi^{x}\left(\mathfrak{s}_{-1}\right)\right)$. Значит, $\psi^{x}: \mathfrak{s}_{-1} \rightarrow T_{x}((M, \mathscr{O}))_{\overline{1}}$ сюръективно тогда и только тогда, когда сюръективно $\mathrm{ev}_{x}: V \rightarrow E_{x}^{*}$. Покажем, что из сюръективности последнего отображения для фиксированной точки $x=o \in M$ вытекает его сюръективность для всех $x \in M$.

Заметим, что расслоение $\mathbf{E}^{*}$ является однородным относительно действия $g \mapsto\left(\Psi(g)^{*}\right)^{-1}$ группы $S_{0}$. В силу (17) для доказательства нашего утверждения достаточно доказать, что $V$ инвариантно относительно $S_{0}$.

Пусть $\mathrm{Ad}$ - присоединенное представление группы $S_{0}$ в $\mathfrak{s}$. Легко видеть, что

$$
\psi((\operatorname{Ad} g) u)=\Psi(g) \psi(u) \Psi(g)^{-1}, \quad g \in S_{0}, \quad u \in \mathfrak{s} .
$$

$\mathrm{C}$ другой стороны, легко проверить, что $\alpha: \Gamma\left(\mathbf{E}^{*}\right) \rightarrow \mathfrak{v}_{-1}(M, \mathscr{O})$ есть изоморфизм $S_{0}$-модулей, если определить в $\mathfrak{v}_{-1}(M, \mathscr{O})$ представление группы $S_{0}$ по формуле

$$
v \mapsto \Psi(g) v \Psi(g)^{-1}, \quad v \in \mathfrak{v}_{-1}(M, \mathscr{O}), \quad g \in S_{0} .
$$

В силу (18) отсюда видно, что $V$ инвариантно.

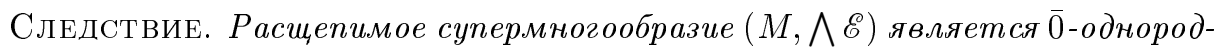
ным тогда и только тогда, когда $\mathbf{E}$ - однородное векторное расслоение. При этом условии $(M, \bigwedge \mathscr{E})$ однородно тогда и только тогда, когда $\mathbf{E}^{*}$ порожда-

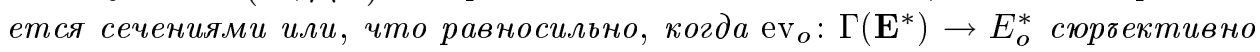
для некоторой фиксированной точки о $\in$ M.

В дальнейшем мы будем рассматривать случай, когда $M$ - флаговое многообразие, т.е. когда $M$ однородно и стабилизаторы $(\operatorname{Bih} M)_{x}^{\circ} \subset(\operatorname{Bih} M)^{\circ}, x \in M$, являются параболическими подгруппами (см. [8], [9]). Тогда группа $\operatorname{Bih} M$ и любая ее транзитивная подгруппа суть полупростые группы Ли. Пусть $\mathbf{E} \rightarrow M-$ однородное векторное расслоение и пусть $Q=\mu\left((\mathrm{Aut} \mathbf{E})^{\circ}\right)$. Тогда $Q$ транзитивна на $M$ и, следовательно, полупроста. Согласно теореме Леви в Aut $\mathbf{E}$ сушествует такая связная подгруппа Ли $G$, что $\mu: G \rightarrow Q$ - локальный изоморфизм. Таким образом, $\mathbf{E}$ однородно относительно связной полупростой группы Ли $G$, локально эффективно действуюшей на $M$.

Из доказанного выше видно, что описание однородных расщепимых супермногообразий $(M, \mathscr{O})$ с флаговым многообразием $M$ сводится к следуюшей задаче. Пусть $M=G / P$, где $G$ - связная полупростая группа Ли, $P$ - ее параболическая подгруппа, не содержащая простых факторов группы $G, \varphi: P \rightarrow \mathrm{GL}(E)$ - голоморфное линейное представление. При каких условиях на $\varphi$ однородное расслоение $\mathbf{E}_{\varphi}$ порождается сечениями? Ответ на этот вопрос дан в работе [10]. Мы сформулируем его в случае, когда $\varphi$ вполне приводимо (доказательство в этом случае, основанное на теореме Бореля-Вейля-Ботта, см. в [6, лемма 7]). 
Введем сначала необходимые понятия и обозначения. Выберем в группе $G$ максимальный тор $T$ и обозначим через $\mathfrak{t}$ соответствуюшую картановскую подалгебру в ее касательной алгебре $\mathfrak{g}$. Пусть $\Delta-$ система корней, связанная с $T$, и пусть выбрана подсистема положительных корней $\Delta_{+} \subset \Delta$. Обозначим через П соответствуюшую систему простых корней.

В $\mathfrak{g}$, как обычно, рассматривается невырожденное $G$-инвариантное скалярное произведение $(\cdot, \cdot)$, индуцируюшее невырожденное скалярное произведение в $\mathfrak{t}^{*}$, инвариантное относительно группы Вейля $W$. Пусть $\left\{h_{\alpha}: \alpha \in \Delta\right\} \subset \mathfrak{t}-$ двойственная к $\Delta$ система корней, тогда $\beta\left(h_{\alpha}\right)=2(\beta, \alpha) /(\alpha, \alpha)$ для любого $\beta \in \mathfrak{t}^{*}$. Элемент $\beta \in \mathfrak{t}^{*}$ называется весом, если $\beta\left(h_{\alpha}\right) \in \mathbb{Z}$ для всех $\alpha \in \Delta$. Вес $\beta$ называется доминантным, если $\beta\left(h_{\alpha}\right) \geqslant 0$ для всех $\alpha \in \Delta_{+}$, и строго доминантным, если $\beta\left(h_{\alpha}\right)>0$ для всех $\alpha \in \Delta_{+}$.

Обозначим через $\mathfrak{g}_{\alpha}$ корневое подпространство в $\mathfrak{g}$, отвечающее корню $\alpha \in \Delta$. Если $R$ - подгруппа Ли в $G$, нормализуемая тором $T$, то ее касательная подалгебра $\mathfrak{r} \subset \mathfrak{g}$ имеет вид

$$
\mathfrak{r}=(\mathfrak{r} \cap \mathfrak{t}) \oplus \bigoplus_{\alpha \in \Delta(R)} \mathfrak{g}_{\alpha},
$$

где $\Delta(R) \subset \Delta$ - замкнутая подсистема, назьваемая системой корней подгруп$n$ b $R$. В частности, выбор системы положительных корней $\Delta_{+}$определяет две борелевские (максимальные разрешимые) подгруппы $B_{ \pm}$в $G$, содержашие $T$, с системами корней $\Delta\left(B_{ \pm}\right)= \pm \Delta_{+}$.

Пусть $P$ - параболическая подгруппа группы $G$, т.е. подгруппа, содержащая борелевскую подгруппу. В дальнейшем мы будем считать, что $P$ содержит подгрупу $B_{-}$, соответствуюшую системе отрицательных корней $\Delta_{-}=-\Delta_{+}$. Как известно (см. [8], [9]), в этом случае $\Delta(P)=\Delta_{-} \cup[\Sigma]$, где $[\Sigma]-$ множество всех корней группы $G$, линейно выражаюшихся через подмножество $\Sigma \subset$ П. Имеется полупрямое разложение

$$
P=H \ltimes N_{-},
$$

где $H$ - максимальная редуктивная подгруппа, а $N_{-}$- унипотентный радикал групшы $P$. При этом

$$
\begin{aligned}
\Delta(H) & =[\Sigma], \\
\Delta\left(N_{-}\right) & =\Delta_{-} \backslash[\Sigma]
\end{aligned}
$$

и $\Sigma$ совпадает с системой простых корней группы $H$, соответствуюшей ее борелевской подг руппе $B_{+} \cap H$. Обозначим через $N_{+}$унипотентную подгруппу с системой корней $\Delta\left(N_{+}\right)=-\Delta\left(N_{-}\right)$. Тогда для соответствуюших алгебр Ли имеем следуюшие разложения:

$$
\begin{aligned}
& \mathfrak{p}=\mathfrak{n}_{-} \boxplus \mathfrak{h}, \\
& \mathfrak{g}=\mathfrak{p}+\mathfrak{n}_{+} \text {(прямая сумма векторных пространств). }
\end{aligned}
$$

Заметим, что $T$ является максимальньм тором и в $P$. Всякий вес представления $\varphi$ группы $P$ является весом в указанном выше смысле. Представление $\varphi$ вполне приводимо тогда и только тогда, когда оно тривиально на $N_{-}$; в этом случае оно полностью определяется представлением $\varphi \mid H$. Старший вес последнего 
представления понимается относительно упорядочения, соответствующего борелевской подгруппе $B_{+} \cap H$. В дальнейшем мы будем писать для краткости $N=N_{-}$, $\mathfrak{n}=\mathfrak{n}_{-}$.

ПРЕДЛОЖЕНИЕ 2. Пусть $\varphi$ вполне приводимо. Тогда следующие свойства эквивалентны:

(1) расслоение $\mathbf{E}_{\varphi}$ порождается сечениями;

(2) все стариие веса представления ч доминантны.

Используя следствие из теоремы 2 , получаем отсюда следующий критерий однородности.

Теорема 3. Пусть $M=G / P$, где $G$ - связная полупростая группа Ли, $P$ - ее параболическая подгруппа, содержащая $B_{-}$, причем $G$ действует на $M$ локально әффективно, $\varphi: P \rightarrow \mathrm{GL}(E)$ - вполне приводимое голоморфное линейное представление. Расщепимое супермногообразие $\left(M, \bigwedge \mathscr{E}_{\varphi}\right)$ однородно тогда и только тогда, когда все старшие веса представления $\varphi^{*}$ доминантHbl.

Опишем строение групшы $G$ и подгруппы $P$ в случае, когда $M=\mathbb{C P}^{n}$. Пусть $\mathbf{E} \rightarrow \mathbb{C P}^{n}-$ однородное векторное расслоение. Как известно, группа Bih $\mathbb{C P}^{n}$ естественно изоморфна проективной групше $\mathrm{PSL}_{n+1}(\mathbb{C})$. Далее, $Q=\mu(\mathrm{Aut} \mathbf{E})$ - подгруппа Ли в $\operatorname{Bih~} \mathbb{C P}^{n}$, транзитивная на $\mathbb{C P}^{n}$. Kaк известно (см. $\left.[9, \S 15]\right)$, возможны следуюшие два случая:

1) $Q=\operatorname{Bih} \mathbb{C P}^{n}=\operatorname{PSL}_{n+1}(\mathbb{C})$;

2) $n=2 r-1$ нечетно и $Q=\operatorname{PSp}_{2 r}(\mathbb{C})=\operatorname{Sp}_{2 r}(\mathbb{C}) /\{E,-E\}$.

В первом случае можно взять в качестве $G$ односвязную группу Ли $\mathrm{SL}_{n+1}(\mathbb{C})$, а во втором - ее подгруппу $\mathrm{Sp}_{2 r}(\mathbb{C})$. При этом можно считать, что $\mu \Phi-$ естественное проективное действие группы $G$ на $\mathbb{C P}^{n}$. В однородных координатах оно задается формулой

$$
(A, u) \mapsto A\left(\begin{array}{c}
u_{1} \\
\vdots \\
u_{n+1}
\end{array}\right), \quad A \in G, \quad u=\left(u_{1}: \ldots: u_{n+1}\right) \in \mathbb{C P}^{n}
$$

В качестве $о$ выберем точку $(0: \cdots: 1)$. Опишем стабилизатор $P=G_{o}$ и соответствующую подалгебру $\mathfrak{p} \subset \mathfrak{g}$.

Пусть сначала $G=\mathrm{SL}_{n+1}(\mathbb{C})$. Тогда $P$ состоит из матрищ вида

$$
\left(\begin{array}{cc}
A & 0 \\
a & \operatorname{det}^{-1} A
\end{array}\right), \quad A \in \mathrm{GL}_{n}(\mathbb{C}), \quad a \in \mathbb{C}^{n} .
$$

Выберем в качестве $T$ подгруппу всех диагональных матриц из $G$. Соответствующая картановская подалгебра $\mathfrak{t} \subset \mathfrak{s l}_{n+1}(\mathbb{C})$ состоит из диагональных матриц

$$
\operatorname{diag}\left(\lambda_{1}, \ldots, \lambda_{n+1}\right), \quad \lambda_{1}+\cdots+\lambda_{n+1}=0
$$

Система корней относительно $T$ имеет вид

$$
\Delta=\left\{\lambda_{i}-\lambda_{j}: i \neq j\right\} .
$$


Положим

$$
\Delta_{+}=\left\{\lambda_{i}-\lambda_{j}: i<j\right\} .
$$

Тогда система простых корней имеет вид

$$
\Pi=\left\{\alpha_{i}=\lambda_{i}-\lambda_{i+1}: i=1, \ldots, n\right\} .
$$

Очевидно, соответствующая подгруппа Бореля $B_{-}-$это подгруппа всех нижних треугольных матриц; она содержится в $P$. Легко видеть также, что

$$
N=\left\{\left(\begin{array}{cc}
E_{n} & 0 \\
a & 1
\end{array}\right)\right\}, \quad H=\left\{\left(\begin{array}{cc}
A & 0 \\
0 & \operatorname{det}^{-1} A
\end{array}\right)\right\} .
$$

Соответствие $A \mapsto\left(\begin{array}{cc}A & 0 \\ 0 & \operatorname{det}^{-1} A\end{array}\right)$ отождествляет $H$ с GL ${ }_{n}(\mathbb{C})$. Кроме того,

$$
\begin{aligned}
& \mathfrak{h}=\left\{\left(\begin{array}{cc}
X & 0 \\
0 & -\operatorname{tr} X
\end{array}\right)\right\} \simeq \mathfrak{g l}_{n}(\mathbb{C}) \\
& \mathfrak{n}=\left\{\left(\begin{array}{cc}
0_{n} & 0 \\
x & 0
\end{array}\right)\right\}
\end{aligned}
$$

Имеем также

$$
\Sigma=\left\{\alpha_{1}, \ldots, \alpha_{n-1}\right\} .
$$

Теперь рассмотрим случай $G=\operatorname{Sp}_{2 r}(\mathbb{C}), n=2 r$. Алгебра Ли $\mathfrak{s p}_{2 r}(\mathbb{C})$ этой групшы есть подалгебра в $\mathfrak{s l}_{2 r}(\mathbb{C})$, состоящая из матриц вида

$$
\left(\begin{array}{cc}
U & V \\
W & -U^{\top}
\end{array}\right), \quad U, V, W \in \mathfrak{g l}_{r}(\mathbb{C}), \quad V^{\top}=V, \quad W^{\top}=W
$$

В этом случае, во избежание путаницы, мы будем обозначать описанные выше подгрупшы $T, P, H, N \subset \mathrm{SL}_{n+1}(\mathbb{C})$ через $\widetilde{T}, \widetilde{P}, \widetilde{H}, \widetilde{N}$, а их алгебры Ли через $\widetilde{\mathfrak{t}}$, $\widetilde{\mathfrak{p}}, \widetilde{\mathfrak{h}}, \widetilde{\mathfrak{n}}$, соответственно. Тогда

$$
P=\widetilde{P} \cap \mathrm{Sp}_{2 r}(\mathbb{C}), \quad \mathfrak{p}=\widetilde{\mathfrak{p}} \cap \mathfrak{s p}_{2 r}(\mathbb{C}),
$$

так что $\mathfrak{p}$ состоит из матриц вида

$$
\left(\begin{array}{cccc}
X & x & Y & 0 \\
0 & b & 0 & 0 \\
Z & z & -X^{\top} & 0 \\
z^{\top} & a & -x^{\top} & -b
\end{array}\right)
$$

где $Y^{\top}=Y, Z^{\top}=Z$.

Подгруппа $T=\widetilde{T} \cap G$ является максимальным тором в $G=\mathrm{Sp}_{2 r}(\mathbb{C})$. Подалгебра Картана $\mathfrak{t}$ в $\mathfrak{s p}_{2 r}(\mathbb{C})$ выделяется в $\widetilde{\mathfrak{t}}$ уравнениями $\lambda_{r+1}=-\lambda_{1}, \ldots, \lambda_{2 r}=-\lambda_{r}$. Введем в $\mathfrak{t}$ координаты

$$
\mu_{1}=\lambda_{r}, \quad \mu_{2}=\lambda_{2}, \ldots, \quad \mu_{r-1}=\lambda_{r-1}, \quad \mu_{r}=\lambda_{1},
$$


являющиеся весами стандартного представления группы $\mathrm{Sp}_{2 r}(\mathbb{C})$. Система корней этой группы имеет вид

$$
\Delta=\left\{\mu_{i}-\mu_{j}, \mu_{i}+\mu_{j}, 2 \mu_{i}: i \neq j\right\}
$$

Выберем в качестве системы положительных корней этой групшы

$$
\Delta_{+}=\left\{\mu_{i}-\mu_{j}, \mu_{i}+\mu_{j}, 2 \mu_{i}: i<j\right\}
$$

Тогда

$$
\Pi=\left\{\beta_{1}=\mu_{1}-\mu_{2}, \ldots, \beta_{r-1}=\mu_{r-1}-\mu_{r}, \beta_{r}=2 \mu_{r}\right\}
$$

Легко проверить, что подалгебра $\mathfrak{p}$ содержит борелевскую подалгебру $\mathfrak{b}_{-}$, отвечающую системе отрицательных корней $\Delta_{-}$. Кроме того, имеем

$$
\begin{aligned}
& \mathfrak{n}=\left\{\left(\begin{array}{cccc}
0 & x & 0 & 0 \\
0 & 0 & 0 & 0 \\
0 & z & 0 & 0 \\
z^{\top} & a & -x^{\top} & 0
\end{array}\right)\right\}, \\
& \mathfrak{h}=\widetilde{\mathfrak{h}} \cap \mathfrak{s p}_{2 r}(\mathbb{C})=\left\{\left(\begin{array}{cccc}
X & 0 & Y & 0 \\
0 & b & 0 & 0 \\
Z & 0 & -X^{\top} & 0 \\
0 & 0 & 0 & -b
\end{array}\right)\right\}, \\
& \Sigma=\left\{\beta_{2}, \ldots, \beta_{r}\right\} .
\end{aligned}
$$

Отметим также, что

$$
\mathfrak{h}=\mathfrak{h}^{\prime} \oplus \mathfrak{z}
$$

где $\mathfrak{h}^{\prime}=[\mathfrak{h}, \mathfrak{h}] \simeq \mathfrak{s p}_{2 r-2}(\mathbb{C})$ выделяется в $\mathfrak{h}$ условием $b=0$, a $\mathfrak{z}=\left\langle y_{0}\right\rangle$, $y_{0}=E_{r r}-E_{2 r, 2 r},-$ одномерный центр в $\mathfrak{h}$. Очевидно,

$$
\mathfrak{r a d} \mathfrak{p}=\mathfrak{n} \boxplus \mathfrak{z}
$$

Обозначая через $N, H, H^{\prime}, Z$ соответствующие связные подгруппы Ли в $G=$ $\mathrm{Sp}_{2 r}(\mathbb{C})$, имеем

$$
H=H^{\prime} \times Z, \quad H^{\prime} \simeq \operatorname{Sp}_{2 r-2}(\mathbb{C}), \quad B \simeq \mathbb{C}^{\times}, \quad \operatorname{Rad} P=N \rtimes Z
$$

При этом $N$ - унипотентный радикал, а $H$ - редуктивная подалгебра в $P$. 


\section{§4. Расщепимые супермногообразия, $\overline{0}$-однородные относительно $\mathrm{SL}_{n+1}(\mathbb{C})$}

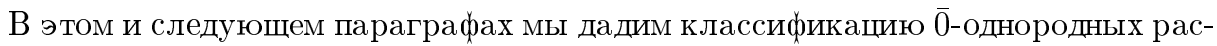
шепимых супермногообразий вида $\left(\mathbb{C P}^{n}, \bigwedge \mathscr{E}\right)$, где нечетная размерность $m \leqslant n$. Будем называть такое супермногообразие $\overline{0}$-однородным относительно $G=$ $\mathrm{SL}_{n+1}(\mathbb{C})$ или $\mathrm{Sp}_{2 r}(\mathbb{C}), 2 r=n+1$, если соответствующее расслоение $\mathbf{E}$ однородно относительно $G$.

Здесь мы рассмотрим случай $G=\mathrm{SL}_{n+1}(\mathbb{C})$. Тогда $P$ задается формулой $(20)$. Опишем представления этой групшы, имеющие размерность $m \leqslant n$. Обозначим через $\rho$ стандартное линейное представление групшы $H=\mathrm{GL}_{n}(\mathbb{C})$ и через $d$ характер $d(A)=\operatorname{det} A$ этой групшы. Теми же буквами обозначим продолжения этих представлений на $P$, равные 1 на $N$.

Лемма 1. Пусть $\varphi: P \rightarrow \mathrm{GL}(E)$ - голоморфное линейное представление размерности $m \leqslant n$. Тогда $\varphi$ вполне приводимо и верно одно из следующих утверждений:

(1) справедливо равенство $m=n u \varphi$ - неприводимое представление, причем либо

$$
\varphi \simeq \rho d^{k}, \quad k \in \mathbb{Z}
$$

либо

$$
\varphi \simeq \rho^{*} d^{k}, \quad k \in \mathbb{Z}
$$

(2) представление ч разлагается в сумму одномерных представлений

$$
\varphi \simeq d^{k_{1}}+\cdots+d^{k_{m}}, \quad k_{i} \in \mathbb{Z}
$$

ДокаЗАТЕЛьство. В случае $n=1$ имеем $m=1$, откуда легко следует, что $\varphi=d^{k}, k \in \mathbb{Z}$. Будем считать, что $m \geqslant 2$. Положим $\mathfrak{h}^{\prime}=[\mathfrak{h}, \mathfrak{h}]$. Тогда нетрудно показать, что $\left[\mathfrak{h}^{\prime}, \mathfrak{n}\right]=\mathfrak{n}$ и, тем более, $[\mathfrak{p}, \mathfrak{n}]=\mathfrak{n}$. Из классификации представлений группы $\mathrm{SL}_{n}(\mathbb{C})\left(\right.$ см. [9], [11]) следует, что при $m \leqslant n$ либо $\varphi\left(H^{\prime}\right)=\{e\}$, либо $n=m$ и $\varphi\left|H^{\prime} \simeq \rho\right| H^{\prime}$ или $\varphi\left|H^{\prime} \simeq \rho^{*}\right| H^{\prime}$, так что $\varphi$ неприводимо.

Пусть $\varphi: P \rightarrow G L(E)$ - неприводимое представление. Рассмотрим $d \varphi$. По теореме Ли существует $E_{\lambda} \subset E, E_{\lambda} \neq 0,-$ весовое подпространство для $\mathfrak{n}$. Поскольку $\mathfrak{n}-$ идеал в $\mathfrak{p}$, имеем $d \varphi(\mathfrak{p})\left(E_{\lambda}\right) \subset E_{\lambda}$ и, следовательно, $E_{\lambda}=E$. Так как $\mathfrak{n}=[\mathfrak{p}, \mathfrak{n}]$, то $\lambda=0$ и, значит, $N \subset \operatorname{Ker} \varphi$. Поскольку $m=n$, отсюда следует, что $\varphi$ имеет вид (23) или (24).

Если $\varphi\left(H^{\prime}\right)=\{e\}$ или $d \varphi\left(\mathfrak{h}^{\prime}\right)=0$, то $d \varphi(\mathfrak{n})=d \varphi\left(\left[\mathfrak{h}^{\prime}, \mathfrak{n}\right]\right)=0$, и $\varphi(N)=\{e\}$. Таким образом, $\varphi$ полностью определяется своим ограничением на $Z\left(\mathrm{GL}_{n}(\mathbb{C})\right) \simeq \mathbb{C}^{\times}$. Отсюда следует, что $\varphi$ имеет вид (25).

Из леммы 1 и теоремы 2 (1) непосредственно вытекает

ТЕОРема 4. Пусть $\left(\mathbb{C P}^{n}, \mathscr{O}\right)$ - расщепимое супермногообразие нечетной размерности $m \leqslant n, \overline{0}$-однородное относительно $\mathrm{SL}_{n+1}(\mathbb{C})$. Тогда $\mathscr{O} \simeq \bigwedge \mathscr{E} \varphi$, 
где $\varphi$ - одно из представлений подгруппь $P \subset \mathrm{SL}_{n+1}(\mathbb{C})$ вида (23), (24) или (25).

Далее мы будем пользоваться картановской подалгеброй (21). Заметим, что веса алгебры Ли $\mathfrak{s l}_{n+1}(\mathbb{C})$ имеют вид

$$
\lambda=\sum_{i=1}^{n+1} a_{i} \lambda_{i}, \quad a_{i} \in \mathbb{Z} .
$$

Вес $\lambda$ доминантен тогда и только тогда, когда $a_{1} \geqslant \cdots \geqslant a_{n+1}$. Старший вес представления $\rho$ (соответственно, $\left.\rho^{*}\right)$ группы $H$ равен $\lambda_{1}\left(\right.$ соответственно, $\left.-\lambda_{n}\right)$, a вес представления $d$ есть $\sum_{i=1}^{n} \lambda_{i}=-\lambda_{n+1}$.

Теорема 5. Расщепимое супермногообразие $\left(\mathbb{C P}^{n}, \bigwedge \mathscr{E}\right)$ нечетной размерности $m \leqslant n, \overline{0}$-однородное относительно $\mathrm{SL}_{n+1}(\mathbb{C})$, однородно тогда и только тогда, когда расслоение $\mathbf{E}$ задается одним из следующих представлений $\varphi$ подгруппы $P \subset \mathrm{SL}_{n+1}(\mathbb{C}):$

(1) $\varphi \simeq \rho d^{k}, \quad k \leqslant-1$;

(2) $\varphi \simeq \rho^{*} d^{k}, \quad k \leqslant 0$;

(3) $\varphi \simeq d^{k_{1}}+\cdots+d^{k_{m}}, \quad k_{i} \leqslant 0$.

ДоказАтЕЛьство. Согласно теореме 4 можно считать, что $\mathbf{E}$ задается представлением $\varphi$ вида $(23),(24)$ или $(25)$, где $k, k_{i} \in \mathbb{Z}$. Тогда

$$
\varphi^{*} \simeq \begin{cases}\rho^{*} d^{-k} & \text { в случае }(23), \\ \rho d^{-k} & \text { в случае }(24), \\ d^{-k_{1}}+\cdots+d^{-k_{m}} & \text { в случае }(25) .\end{cases}
$$

Согласно теореме 3 супермногообразие $\left(\mathbb{C P}^{n}, \bigwedge \mathscr{E}\right)$ однородно тогда и только тогда, ког да все старшие веса $\Lambda^{*}$ этого представления доминантны. Очевидно,

$$
\Lambda^{*}= \begin{cases}-\lambda_{n}+k \lambda_{n+1} & \text { в случае }(23), \\ \lambda_{1}+k \lambda_{n+1} & \text { в случае }(24), \\ k_{i} \lambda_{n+1}, i=1, \ldots, m, & \text { в случае }(25) .\end{cases}
$$

Отсюда следует наше утверждение.

ЗАмЕчАнИЕ. Как видно из теоремы 3 , представление $\varphi$ вида (3) определяет однородное расшепимое супермногообразие при любом $m \geqslant 1$.

\section{§5. Расщепимые супермногообразия, $\overline{0}$-однородные относительно $\mathrm{Sp}_{2 r}(\mathbb{C})$}

В этом параграфе рассматривается случай $G=\operatorname{Sp}_{2 r}(\mathbb{C}), 2 r=n+1 \geqslant 4$. Мы пользуемся обозначениями, введенными в $\S 3$. Начнем с классификации представлений $\varphi: P \rightarrow \mathrm{GL}_{m}(\mathbb{C}), m \leqslant n$. Очевидно, расслоение $\mathbf{E}_{\varphi}$ однородно относительно $\mathrm{Sp}_{2 r}(\mathbb{C})$, но не относительно $\mathrm{SL}_{n+1}(\mathbb{C})$, тог да и только тогда, ког да $\varphi$ не продолжается до представления $\widetilde{P} \rightarrow \mathrm{GL}_{m}(\mathbb{C})$. Нам достаточно перечислить только такие представления. 
Обозначим через $\rho$ стандартное представление групшы $H^{\prime} \simeq \mathrm{Sp}_{2 r-2}(\mathbb{C})$, через 1 - тривиальное одномерное представление и через $d-$ характер групшы $Z \simeq \mathbb{C}^{\times}$, заданный формулой

$$
d\left(\begin{array}{cccc}
E & 0 & 0 & 0 \\
0 & b & 0 & 0 \\
0 & 0 & E & 0 \\
0 & 0 & 0 & b^{-1}
\end{array}\right)=b
$$

Для любого голоморфного представления $\psi$ редуктивной группы $H$ (которое, как известно, вполне приводимо, см. $[11, \S 5.2])$ будем обозначать тем же символом $\psi$ его продолжение на $P$, равное 1 на $N$.

Лемма 2. Пусть $\varphi: P \rightarrow \mathrm{GL}_{m}(E), m \leqslant n,-$ голоморфное линейное представление, не продоләсающееся до представления $\widetilde{P} \rightarrow \mathrm{GL}_{m}($ E). Тогда $\varphi$ вполне приводимо и верно одно из следующих утверждений:

(1) $\varphi \simeq \rho \otimes d^{k}, k \in \mathbb{Z}$;

(2) $\varphi \simeq \rho \otimes d^{k}+1 \otimes d^{l}, k, l \in \mathbb{Z}$;

(3) $n=3, \varphi \simeq \sigma \otimes d^{k}, k \in \mathbb{Z}$, әде $\sigma-$ трехмерное неприводимое представление группь $\mathrm{Sp}_{2}(\mathbb{C})=\mathrm{SL}_{2}(\mathbb{C}) ;$

(4) $n=5, \varphi \simeq \nu \otimes d^{k}, k \in \mathbb{Z}$, где $\nu$ - пятимерное неприводимое представление группьл $\mathrm{Sp}_{4}(\mathbb{C})$.

ДокАЗАТЕЛЬСтво. Пусть $\mathfrak{a}=\left\langle E_{2 r, r}\right\rangle$, тогда $\mathfrak{n}=\mathfrak{m} \oplus \mathfrak{a}$ (прямая сумма векторных пространств), где $\mathfrak{m} \simeq \mathbb{C}^{2 r-2}$. Положим также $\mathfrak{f}=\mathfrak{z} \oplus \mathfrak{a}$, тогда $\mathfrak{r a d} \mathfrak{p}=\mathfrak{z} \oplus \mathfrak{m}$ (прямые суммы векторных пространств). Нетрудно проверить, что верны соотношения:

$$
\begin{gathered}
{\left[\mathfrak{h}^{\prime}, \mathfrak{m}\right]=\mathfrak{m}} \\
{[\mathfrak{m}, \mathfrak{m}]=\mathfrak{a}} \\
{[\mathfrak{a}, \mathfrak{m}]=0} \\
{\left[\mathfrak{h}^{\prime}, \mathfrak{r a d} \mathfrak{p}\right]=\mathfrak{m}, \quad[\mathfrak{r} \mathfrak{a} \mathfrak{d} \mathfrak{p}, \mathfrak{r a d} \mathfrak{p}]=\mathfrak{n} .}
\end{gathered}
$$

1) Пусть $m<n-1=2 r-2$, тогда, как известно из теории представлений (см., например, [9, предложение 1.3.5]), $d \varphi\left(\mathfrak{h}^{\prime}\right)=0$. Используя (30) и (31), получаем $d \varphi(\mathfrak{m})=d \varphi(\mathfrak{a})=0$. Следовательно, $\varphi \simeq 1 \otimes\left(d^{k_{1}}+\cdots+d^{k_{m}}\right), k_{i} \in \mathbb{Z}$. Но ясно, что $1 \otimes d$ продолжается до представления $\operatorname{det}$ групшы $\widetilde{P}$, так что $\varphi$ также продолжается на $\widetilde{P}$.

2) Пусть $m=n-1=2 r-2$, тогда если $d \varphi\left(\mathfrak{h}^{\prime}\right)=0$, то все рассуждения совпадают со случаем 1$)$. Если $d \varphi\left(\mathfrak{h}^{\prime}\right) \neq 0$, то из теории представлений следует, что $d \varphi \mid \mathfrak{h}^{\prime} \simeq \rho$, и $\varphi$ неприводимо. Пусть $x \in \mathfrak{r a d} \mathfrak{p}$, тогда

$$
d \varphi(x)=\lambda(x) E
$$

где $\lambda: \mathfrak{r a d} \mathfrak{p} \rightarrow \mathbb{C}$. Как известно, $\lambda([\mathfrak{p}, \mathfrak{r a d} \mathfrak{p}])=0$. В силу $(33)$

$$
[\mathfrak{p}, \mathfrak{r a d} \mathfrak{p}]=\left[\mathfrak{h}^{\prime}, \mathfrak{r a d} \mathfrak{p}\right]+[\mathfrak{r a d} \mathfrak{p}, \mathfrak{r a d} \mathfrak{p}]=\mathfrak{n}
$$


откуда следует, что $d \varphi(\mathfrak{n})=0$. Таким образом, $\varphi$ определяется своим ограничением на $H$. Значит, $\varphi$ вполне приводимо и имеет вид $(26)$.

3) Пусть $m=n=2 r-1$, тогда если $d \varphi\left(\mathfrak{h}^{\prime}\right)=0$, то все рассуждения такие же, как и в случае 1$)$. Пусть $d \varphi\left(\mathfrak{h}^{\prime}\right) \neq 0$, тогда возможны следующие случаи (см., например, $[9$, предложение 1.3.5]):

(a) $d \varphi \mid \mathfrak{h}^{\prime} \simeq d \rho+0$

(b) $n=3, d \varphi \mid \mathfrak{h}^{\prime} \simeq d \sigma$ - трехмерное неприводимое представление;

(c) $n=5, d \varphi \mid \mathfrak{h}^{\prime} \simeq d \nu$-пятимерное неприводимое представление.

В случае (a) пространство представления $E$ можно разложить в прямую сумму инвариантных подпространств

$$
E=W_{1} \oplus W_{0}
$$

где $\operatorname{dim} W_{1}=2 r-2, \operatorname{dim} W_{0}=1$. Тогда ясно, что

$$
\varphi \mid H \simeq \rho \otimes d^{k}+1 \otimes d^{l}, \quad k, l \in \mathbb{Z}
$$

Если $d \varphi(\mathfrak{n})=0$, то $\varphi$ вполне приводимо и имеет место (27). Предположим, что $d \varphi(\mathfrak{n}) \neq 0$. Ограничение $d \varphi \mid \mathfrak{r a d} \mathfrak{p}$ имеет весовое подпространство $E_{\lambda} \neq 0$, где $\lambda(\mathfrak{n})=0$, инвариантное относительно $\mathfrak{p}$. Возможны случаи $E_{\lambda}=W_{1}, E_{\lambda}=W_{0}$. Пусть $E_{\lambda}=W_{1}$. Тогда для $y \in \mathfrak{h}^{\prime}, x \in \mathfrak{n}$ операторы представления записываются в подходяшем базисе матрицами

$$
\begin{aligned}
& d \varphi(y)=\left(\begin{array}{cc}
\rho(y) & 0 \\
0 & 0
\end{array}\right), \\
& d \varphi(x)=\left(\begin{array}{cc}
0 & \alpha(x) \\
0 & 0
\end{array}\right),
\end{aligned}
$$

где $\alpha: \mathfrak{n} \rightarrow \mathbb{C}^{2 r-2}-$ ненулевое линейное отображение. Если $z=y+b y_{0} \in \mathfrak{h}, y \in \mathfrak{h}^{\prime}$, $b \in \mathbb{C}$, то

$$
d \varphi(z)=\left(\begin{array}{cc}
y+k b & 0 \\
0 & l b
\end{array}\right)
$$

Если $x_{1}, x_{2} \in \mathfrak{n}$, то

$$
d \varphi\left(\left[x_{1}, x_{2}\right]\right)=\left[d \varphi\left(x_{1}\right), d \varphi\left(x_{2}\right)\right]=\left[\left(\begin{array}{cc}
0 & \alpha\left(x_{1}\right) \\
0 & 0
\end{array}\right),\left(\begin{array}{cc}
0 & \alpha\left(x_{2}\right) \\
0 & 0
\end{array}\right)\right]=0 .
$$

Но в то же время

$$
d \varphi\left(\left[x_{1}, x_{2}\right]\right)=\left(\begin{array}{cc}
0 & \alpha\left(\left[x_{1}, x_{2}\right]\right) \\
0 & 0
\end{array}\right) .
$$

В силу (21) и (22) отсюда следует, что $d \varphi(\mathfrak{a})=0$. Для любых $y \in \mathfrak{h}^{\prime}, x \in \mathfrak{n}$ имеем $[y, x] \in \mathfrak{n}$, откуда

$$
d \varphi([y, x])=\left(\begin{array}{cc}
0 & \alpha([y, x]) \\
0 & 0
\end{array}\right)
$$


С другой стороны,

$$
d \varphi([y, x])=[d \varphi(y), d \varphi(x)]=\left[\left(\begin{array}{ll}
y & 0 \\
0 & 0
\end{array}\right),\left(\begin{array}{cc}
0 & \alpha(x) \\
0 & 0
\end{array}\right)\right]=\left(\begin{array}{cc}
0 & y \alpha(x) \\
0 & 0
\end{array}\right) .
$$

Получили равенство

$$
\alpha([y, x])=y \alpha(x), \quad y \in \mathfrak{h}^{\prime}, \quad x \in \mathfrak{n} .
$$

Поскольку $\alpha \neq 0$, а $\rho$ неприводимо, то $\alpha: \mathfrak{n} \rightarrow \mathbb{C}^{2 r-2}$ - сюръективное отображение с ядром Ker $\alpha=\mathfrak{a}$, определяющее изоморфизм $\mathfrak{h}^{\prime}$-модулей $\mathfrak{m} \rightarrow \mathbb{C}^{2 r-2}$.

Легко видеть, что

$$
\left[y_{0}, x\right]=-x, \quad x \in \mathfrak{m} .
$$

Поэтому

$$
d \varphi(-x)=\left(\begin{array}{cc}
0 & -\alpha(x) \\
0 & 0
\end{array}\right)=\left[\left(\begin{array}{cc}
k & 0 \\
0 & l
\end{array}\right),\left(\begin{array}{cc}
0 & \alpha(x) \\
0 & 0
\end{array}\right)\right]=\left(\begin{array}{cc}
0 & (k-l) \alpha(x) \\
0 & 0
\end{array}\right), \quad x \in \mathfrak{m} .
$$

Отсюда $l=k+1$. Теперь нетрудно убедиться в том, что $\varphi$ изоморфно ограничению на $P$ представления $\rho d^{k}$ подг руппы $\widetilde{P} \subset \mathrm{SL}_{n+1}(\mathbb{C})$ (см. лемму 1$)$.

Если $d \varphi \mid \mathfrak{n} \neq 0$ и $E_{\lambda}=W_{0}$, то можно рассмотреть представление $\varphi^{*}$. Тогда $W_{0}^{\perp} \subset E^{*}$ является $(2 r-2)$-мерным весовым подпространством для $d \varphi \mid \mathfrak{r a d} \mathfrak{p}$, так что в силу доказанного выше $\varphi^{*}$ продолжается на $\widetilde{P}$. Значит, это верно и для $\varphi$. В случаях (b) и (c) рассуждения проводятся так же, как в пункте 2).

Из леммы 2 вытекает

Теорема 6. Пусть $\left(\mathbb{C P}^{n}, \mathscr{O}\right)$ - расщепимое супермногообразие нечетной размерности $m \leqslant n=2 r-1, \overline{0}$-однородное относительно $\operatorname{Sp}_{2 r}(\mathbb{C})$, но не относительно $\mathrm{SL}_{n+1}(\mathbb{C})$. Тогда $\mathscr{O} \simeq \bigwedge \mathscr{E}_{\varphi}$, где $\varphi$ - одно из представлений подгруппь $P \subset \mathrm{Sp}_{2 r}(\mathbb{C})$ вида (26), (27), (28) или (29).

Будем пользоваться картановской подалгеброй $\mathfrak{t}=\tilde{\mathfrak{t}} \cap \mathfrak{s p}_{2 r}(\mathbb{C})$. Заметим, что веса алгебры Ли $\mathfrak{s p}_{2 r}(\mathbb{C})$ имеют вид

$$
\lambda=\sum_{i=1}^{r} a_{i} \mu_{i}, \quad a_{i} \in \mathbb{Z}
$$

Вес $\lambda$ доминантен тогда и только тогда, когда $a_{1} \geqslant \cdots \geqslant a_{r} \geqslant 0$. Вес представления $d$ равен $\mu_{1}=\lambda_{r}$, а старший вес представления $\rho$ группы $H^{\prime}$ равен $\mu_{2}=\lambda_{2}$.

Теорема 7. Пусть $\varphi: P \rightarrow \mathrm{GL}_{m}(E), m \leqslant n,-$ голоморфное линейное представление, не продолжающееся до представления $\widetilde{P} \rightarrow \mathrm{GL}_{m}(E)$. Расщепимое супермногообразие, определенное таким представлением, однородно тогда и только тогда, когда

(1) $\varphi \simeq \rho \otimes d^{k}, \quad k \leqslant-1$

(2) $\varphi \simeq \rho \otimes d^{k}+1 \otimes d^{l}, \quad k \leqslant-1, l \leqslant 0$;

(3) $\varphi \simeq \sigma \otimes d^{k}, \quad k \leqslant-2$;

(4) $\varphi \simeq \nu \otimes d^{k}, k \leqslant-1$. 
ДокАЗАТЕЛЬство. Из теоремы 6 следует, что $\varphi$ имеет вид (26), (27), (28) или (29). Тогда

$$
\varphi^{*} \simeq \begin{cases}\rho \otimes d^{-k} & \text { в случае }(26), \\ \rho \otimes d^{-k}+1 \otimes d^{-l} & \text { в случае (27), } \\ \sigma \otimes d^{-k} & \text { в случае (28), } \\ \nu \otimes d^{-k} & \text { в случае }(29) .\end{cases}
$$

Старшие веса $\Lambda^{*}$ таких представлений имеют вид

$$
\begin{aligned}
& \Lambda^{*}=-k \mu_{1}+\mu_{2} \quad \text { в случае (26), } \\
& \Lambda^{*}=\left\{\begin{array}{l}
-k \mu_{1}+\mu_{2} \\
-l \mu_{1}
\end{array} \quad\right. \text { в случае (27), } \\
& \Lambda^{*}=-k \mu_{1}+2 \mu_{2} \quad \text { в случае (28), } \\
& \Lambda^{*}=-k \mu_{1}+\mu_{2}+\mu_{3} \text { в случае (29). }
\end{aligned}
$$

Согласно теореме 3 супермногообразие $\left(\mathbb{C P}^{n}, \bigwedge \mathscr{E}_{\varphi}\right)$ однородно тогда и только тогда, когда вес $\Lambda^{*}$ доминантен. Отсюда следует наша теорема.

\section{§6. Когомологии касательного пучка}

В этом параграфе мы сделаем первый шаг в направлении классификации четно-однородных и однородных нерасщепимых супермногообразий вида $\left(\mathbb{C} \mathbb{P}^{n}, \mathscr{O}\right)$, нечетная размерность $m$ которых удовлетворяет условию $m \leqslant n$. В силу следствия из теоремы 1 из однородности ( $\overline{0}$-однородности) супермногообразия $\left(\mathbb{C P}^{n}, \mathscr{O}\right)$ следует однородность (соответственно, $\overline{0}$-однородность) его ретракта $\left(\mathbb{C P}^{n}, \mathscr{O}_{\mathrm{gr}}\right)$. Поэтому наша задача будет решена, если для каждого из супермногообразий $\left(\mathbb{C P}^{n}, \mathscr{O}_{\text {gr }}\right)$, перечисленных в $\S 4$ и $\S 5$, мы сможем классифицировать все $\overline{0}$-однородные и однородные супермногообразия, ретрактом которых является $\left(\mathbb{C P}^{n}, \mathscr{O}_{\text {gr }}\right)$. Как мы увидим в части II (см. также [2]), для получения этой классификации весьма существенно выгисление $G$-инвариантных частей групп когомологий $H^{1}\left(\mathbb{C P}^{n}, \mathscr{T}_{2 p}\right)$, где $G=\mathrm{SL}_{n+1}(\mathbb{C})$ или $\mathrm{Sp}_{2 r}(\mathbb{C}), \mathscr{T}$ - касательный пучок супермногообразия $\left(\mathbb{C P}^{n}, \mathscr{O}_{\mathrm{gr}}\right)$ и $p \geqslant 1$. Несомненно, большой интерес представляет и вычисление всех групп когомологий $H^{q}\left(\mathbb{C P}^{n}, \mathscr{T}_{p}\right)$, но мы сможем найти полное решение этой задачи только в некоторых специальных случаях.

Мы будем использовать известную теорему Ботта (см. [8], [12]), при помощи которой можно вычислить градуированное пространство когомологий $H^{*}(M, \mathscr{E})$, где $M=G / P$ - флаговое многообразие, а $\mathscr{E}$ - пучок голоморфных сечений однородного векторного расслоения $\mathbf{E} \rightarrow M$, определяемого неприводимым (или вполне приводимым) представлением $\varphi: P \rightarrow \mathrm{GL}(E)$.

Мы пользуемся обозначениями $\S 3$. Вес $\lambda \in \mathfrak{t}^{*}$ называется сингулярным, если сушествует корень $\alpha \in \Delta_{+}$такой, что $\lambda\left(h_{\alpha}\right)=0$, и регулярнылм в противном случае. Положим $\gamma=\frac{1}{2} \sum_{\alpha \in \Delta_{+}} \alpha$, тогда $\gamma\left(h_{\alpha}\right)=1$ для всех $\alpha \in \Pi$ П. Если $\lambda$ - регулярный вес, то существует единственный $w \in W$ такой, что $w(\lambda)-\gamma$ доминантен. Минимальное число отражений $s_{\alpha}, \alpha \in \Pi$, в произведение которых разлагается элемент $w$, назьвается индексом регулярного веса $\lambda$ (индекс равен 
также числу таких $\alpha \in \Delta_{+}$, что $\left.\lambda\left(h_{\alpha}\right)<0\right)$. С каждым весом $\lambda \in \mathfrak{t}^{*}$ таким, что $\lambda+\gamma$ регулярен, свяжем доминантный вес $I(\lambda) \in \mathfrak{t}^{*}$ по правилу $I(\lambda)=w(\lambda+\gamma)-\gamma$.

Переходя к формулировке теоремы Ботта, заметим, что в каждом пространстве когомологий $H^{q}(M, \mathscr{E})$, связанном с однородньп векторным расслоением $\mathbf{E} \rightarrow M=G / P$, индуцируется естественная структура $G$-модуля (в случае $q=0$ эта структура рассматривалась в $\S 3$ ).

ТЕОРЕма БоТТА. Пусть $\varphi$ - неприводимое конечномерное голоморфное представление подгруппь $P$ со старшим весом $\Lambda$. Тогда градуированное пространство когомологий $H^{*}\left(M, \mathscr{E}_{\varphi}\right)$ определяется по $\Lambda$ следующим образом:

а) если $\Lambda+\gamma$ сингулярен, то $H^{*}\left(M, \mathscr{E}_{\varphi}\right)=0$;

b) если $\Lambda+\gamma$ регулярен и его индекс равен $p$, то $H^{q}\left(M, \mathscr{E}_{\varphi}\right)=0$ для всех $q \neq p$ и $H^{p}\left(M, \mathscr{E}_{\varphi}\right)$ есть неприводимьй $G$-модуль со стариим весом $I(\Lambda)$.

В частности, для $H^{q}\left(M, \mathscr{E}_{\varphi}\right), q=0,1$, получаем следуюшее утверждение (для $q=0$ это так называемая теорема Бореля-Вейля-Ботта, используемая при доказательстве предложения 2). Мы обозначаем через $R(\lambda)$ неприводимое представление группы $G$ со старшим весом $\lambda$ и через $\Sigma$ - подсистему в $\Pi$, определяюшую параболическую подгруппу $P$.

СЛЕДСТВИЕ. Пространство $H^{0}\left(M, \mathscr{E}_{\varphi}\right)=\Gamma\left(\mathbf{E}_{\varphi}\right)$ отлично от 0 тогда и только тогда, когда вес $\Lambda$ доминантен. При этом условии в нем индуцируется представление $R(\Lambda)$.

Пространство $H^{1}\left(M, \mathscr{E}_{\varphi}\right)$ отлично от 0 тогда и только тогда, когда существует такой $\alpha \in \Pi \backslash \Sigma$, что вес $s_{\alpha}(\Lambda)-\alpha$ доминантен. При этом условии такой корень $\alpha$ является единственным и в $H^{1}\left(M, \mathscr{E}_{\varphi}\right)$ индуцируется представление $R\left(s_{\alpha}(\Lambda)-\alpha\right)$.

ДокАЗАТЕЛЬСТво. В случае $q=0$ мы должны иметь $w=e$ и тем самым $I(\Lambda)=\Lambda$.

В случае $q=1$ нужно, чтобы вес $\Lambda+\gamma$ имел индекс 1 , т.е. чтобы $I(\Lambda)=$ $s_{\alpha}(\Lambda+\gamma)-\gamma$ был доминантным весом для некоторого $\alpha \in \Pi$, которьй определяется единственным образом. Очевидно, $I(\Lambda)=s_{\alpha}(\Lambda)+s_{\alpha}(\gamma)-\gamma=s_{\alpha}(\Lambda)-\alpha$. Поскольку $\Lambda$ доминантен как вес подгрупшы $H$ с системой простых корней $\Sigma$, для любого $\beta \in \Sigma$ имеем $I(\Lambda)\left(h_{\beta}\right)=-(\Lambda+\gamma)\left(h_{\beta}\right)-1<0$, что неверно для $\beta=\alpha$. Значит, $\alpha \in \Pi \backslash \Sigma$.

Пусть теперь $\left(\mathbb{C P}^{n}, \bigwedge \mathscr{E}_{\varphi}\right)-\overline{0}$-однородное относительно $\mathrm{SL}_{n+1}(\mathbb{C})$ расщепимое супермногообразие размерности $n \mid m, n \geqslant m$, и $\varphi$-представление, определяющее соответствующее однородное векторное расслоение. Изучение когомологий касательного пучка $\mathscr{T}$ мы начнем с изучения когомологий пучков $\mathscr{A}_{r}=\mathscr{E}_{\varphi}^{*} \otimes \bigwedge^{r} \mathscr{E}_{\varphi}$ и $\mathscr{B}_{r}=\Theta \otimes \bigwedge^{r} \mathscr{E}_{\varphi}$, входящих в точную последовательность (7). Имеем $\Theta=\mathscr{E}_{\tau}$, где $\tau$ - представление изотропии в касательном пространстве к $\mathbb{C P}^{n}$. Как известно, оно неприводимо, и в обозначениях $\S 4$

$$
\tau \simeq \rho d
$$


Возможные представления $\varphi$ указаны в теореме 4 . Следовательно, $\mathscr{A}_{r}$ и $\mathscr{B}_{r}$ определяются вполне приводимыми представлениями

$$
\begin{aligned}
& \mathrm{A}_{r}=\varphi^{*} \bigwedge_{r}^{r} \varphi, \\
& \mathrm{B}_{r}=\tau \bigwedge^{r} \varphi
\end{aligned}
$$

подгруппы $P$ и для вычисления когомологий можно применить теорему Ботта.

В приведенных ниже предложениях 3 и 4 предполагается, что $\varphi$ - представление группы $P$ одного из следующих трех видов:

$$
\begin{gathered}
\text { (I) }: \varphi=\rho d^{k}, \quad k \in \mathbb{Z}, \\
\text { (II) }: \varphi=\rho^{*} d^{k}, \quad k \in \mathbb{Z}, \\
\text { (III) }: \varphi=\sum_{i=1}^{m} d^{k_{i}}, \quad k_{i} \in \mathbb{Z} .
\end{gathered}
$$

Нам нужно разложить представления $\mathrm{A}_{r}$ и $\mathrm{B}_{r}$ на неприводимые компоненты. Будем обозначать через $R(\lambda)$ неприводимое представление группы $\mathrm{SL}_{n+1}(\mathbb{C})$ со старшим весом $\lambda$. Используя известное разложение

$$
\rho \bigwedge \rho^{*}= \begin{cases}\rho & \text { при } r=0, \\ R\left(\lambda_{1}-\lambda_{n-r+1}-\cdots-\lambda_{n}\right)+\bigwedge^{r-1} \rho^{*} & \text { при } 1 \leqslant r \leqslant n-1, \\ \bigwedge^{n-1} \rho^{*} & \text { при } r=n\end{cases}
$$

и изоморфизм

$$
\bigwedge^{r} \rho \simeq\left(\bigwedge^{n-r} \rho^{*}\right) d
$$

легко получаем

ПРЕДЛОЖЕНИЕ 3. Предположим, что $\varphi$ - представление одного из видов (I), (II), (III), указанных в (35). Тогда представления $\mathrm{A}_{r}$ и $\mathrm{B}_{r}$ разлагаются, соответственно, на неприводимые компоненты со следующими старшими весами:

(I) $\mathrm{A}_{r}:-\lambda_{n}+k \lambda_{n+1} \quad(r=0)$,

$$
\begin{aligned}
& (r=1), \\
& \lambda_{1}+\cdots+\lambda_{r}-\lambda_{n}-k(r-1) \lambda_{n+1} \quad(1 \leqslant r \leqslant n-1), \\
& \lambda_{1}+\cdots+\lambda_{r-1}-k(r-1) \lambda_{n+1} \quad(2 \leqslant r \leqslant n) ; \\
\mathrm{B}_{r}: & -\lambda_{r+2}-\ldots-\lambda_{n}-(k r+2) \lambda_{n+1} \quad(0 \leqslant r \leqslant n-2), \\
& \lambda_{1}-\lambda_{r+1}-\ldots-\lambda_{n}-(k r+2) \lambda_{n+1} \quad(1 \leqslant r \leqslant n-1), \\
& -(k(n-1)+2) \lambda_{n+1} \quad(r=n-1), \\
& \lambda_{1}-(k n+2) \lambda_{n+1} \quad(r=n) ;
\end{aligned}
$$


(II) $\mathrm{A}_{r}: \lambda_{1}+k \lambda_{n+1} \quad(r=0)$,

$$
\begin{aligned}
& 0 \quad(r=1), \\
& \lambda_{1}-\lambda_{n-r+1}-\ldots-\lambda_{n}-k(r-1) \lambda_{n+1} \quad(1 \leqslant r \leqslant n-1), \\
& -\lambda_{n-r+2}-\ldots-\lambda_{n}-k(r-1) \lambda_{n+1} \quad(2 \leqslant r \leqslant n) ;
\end{aligned}
$$

$\mathrm{B}_{r}: \lambda_{1}-\lambda_{n+1} \quad(r=0)$,

$-(k+1) \lambda_{n+1} \quad(r=1)$,

$\lambda_{1}-\lambda_{n-r+1}-\ldots-\lambda_{n}-(k r+1) \lambda_{n+1} \quad(1 \leqslant r \leqslant n-1)$,

$-\lambda_{n-r+2}-\ldots-\lambda_{n}-(k r+1) \lambda_{n+1} \quad(2 \leqslant r \leqslant n)$;

(III) $\mathrm{A}_{r}: k_{j} \lambda_{n+1}, j=1, \ldots, m \quad(r=0)$,

$$
\left(-k_{i_{1}}-\ldots-k_{i_{r}}+k_{j}\right) \lambda_{n+1}, i_{1}<\cdots<i_{r}, j=1, \ldots, m(1 \leqslant r \leqslant m) ;
$$

$\mathrm{B}_{r}: \lambda_{1}-\lambda_{n+1} \quad(r=0)$,

$$
\lambda_{1}-\left(1+k_{i_{1}}+\cdots+k_{i_{r}}\right) \lambda_{n+1}, i_{1}<\cdots<i_{r}, j=1, \ldots, m(1 \leqslant r \leqslant m) .
$$

Как мы видели в $\S 3$, в нашем случае подгруппа $P \subset \mathrm{SL}_{n+1}(\mathbb{C})$ определяется системой $\Sigma=\Pi \backslash\left\{\alpha_{n}\right\}$, где $\alpha_{n}=\lambda_{n}-\lambda_{n+1}$. Согласно следствию из теоремы Ботта для вычисления 1-когомологий пучков $\mathscr{A}_{r}$ и $\mathscr{B}_{r}$ мы должны найти те веса $\Lambda$ из списка предложения 3 , для которых вес $s_{\alpha_{n}}(\Lambda)-\alpha_{n}$ доминантен. Проделав несложные вычисления, мы получим следуюшее предложение, описывающее эти когомологии.

ПРЕДЛОЖЕНИЕ 4. Предположим, что $\varphi$ имеет вид (35). В случаях (I) $u$ (II) все возможные ненулевые значения для $H^{1}\left(\mathbb{C P}^{n}, \mathscr{S}\right), \mathscr{I}=\mathscr{A}_{r}, \mathscr{B}_{r}$, указань в следующей таблице, где через $\Phi_{1}$ обозначено представление группь $G=\mathrm{SL}_{n+1}(\mathbb{C})$, индуцированное в $H^{1}\left(\mathbb{C P}^{n}, \mathscr{S}\right)$.

\begin{tabular}{|c|c|c|c|l|}
\hline & $\varphi$ & $n$ & $\mathscr{S}$ & $\Phi_{1}$ \\
\hline 1 & $\rho d$ & $\geqslant 2$ & $\mathscr{A}_{0}$ & $R(0)$ \\
\hline 2 & $\rho d^{-1}$ & $\geqslant 3$ & $\mathscr{A}_{2}$ & $R\left(\lambda_{1}+\lambda_{2}\right)$ \\
\hline 3 & $\rho^{*} d^{-1}$ & $\geqslant 2$ & $\mathscr{A}_{2}$ & $R(0)$ \\
\hline 4 & $\rho^{*} d^{-1}$ & $\geqslant 2$ & $\mathscr{B}_{2}$ & $R(0)$ \\
\hline 5 & $\rho^{*} d^{-2}$ & $\geqslant 2$ & $\mathscr{B}_{1}$ & $R\left(\lambda_{1}\right)$ \\
\hline 6 & $\rho d^{-1}$ & 5 & $\mathscr{B}_{3}$ & $R(0)$ \\
\hline 7 & $\rho d^{-1}$ & 4 & $\mathscr{A}_{3}$ & $R\left(-\lambda_{5}\right)$ \\
\hline 8 & $\rho d^{-1}$ & 4 & $\mathscr{B}_{3}$ & $R\left(\lambda_{1}\right)$ \\
\hline 9 & $\rho d^{-1}$ & 3 & $\mathscr{A}_{3}$ & $R(0)$ \\
\hline 10 & $\rho d^{-2}$ & 3 & $\mathscr{A}_{2}$ & $R\left(-\lambda_{4}\right)$ \\
\hline 11 & $\rho d^{-3}$ & 3 & $\mathscr{B}_{1}$ & $R(0)$ \\
\hline 12 & $\rho d^{-4}$ & 2 & $\mathscr{B}_{1}$ & $R\left(-\lambda_{3}\right)$ \\
\hline
\end{tabular}


B случае (III) имеем $H^{1}\left(\mathbb{C P}^{n}, \mathscr{A}_{r}\right)=0$ при $n \geqslant 2, r \geqslant 0$ и $H^{1}\left(\mathbb{C P}^{n}, \mathscr{B}_{r}\right)=0$ при $n \geqslant 3, r \geqslant 0$ или $n \geqslant 2, r=0$. Неприводимье компоненты $G$-модулей $H^{1}\left(\mathbb{C P}^{2}, \mathscr{B}_{r}\right), r \geqslant 1$, имеют следующий вид: каждому набору $i_{1}, \ldots, i_{r}$ такому, что $1 \leqslant i_{1}<\cdots<i_{r} \leqslant m u k_{i_{1}}+\cdots+k_{i_{r}}=-3$, отвечает тривиальная одномерная компонента в $H^{1}\left(\mathbb{C P}^{2}, \mathscr{B}_{r}\right)$.

Применим этот результат к вычислению инвариантных 1-когомологий касательного пучка.

TЕОРема 8. Пусть $\left(\mathbb{C P}^{n}, \bigwedge \mathscr{E}\right)$ - расщепимое супермногообразие размерносmи $n \mid m, n \geqslant 2, n \geqslant m, \overline{0}$-однородное относительно $\mathrm{SL}_{n+1}(\mathbb{C})$, и $\varphi-$ - педставление, определяющее соответствующее однородное векторное расслоение. Тогда пространство $G$-инвариантов $H^{1}\left(\mathbb{C P}^{n}, \mathscr{T}_{\overline{0}}\right)^{G}$, где $G=\mathrm{SL}_{n+1}(\mathbb{C})$ или $\mathrm{Sp}_{2 r}(\mathbb{C})$, нетривиально в точности в следующих случаях:

(1) $n=m \geqslant 2 u \varphi=\rho^{*} d^{-1}$

(2) $n=m=2 u \varphi=d^{k}+d^{l}$, где $k+l=-3$.

В этих случаях

$$
H^{1}\left(\mathbb{C P}^{n}, \mathscr{T}_{\overline{0}}\right)^{G}=H^{1}\left(\mathbb{C P}^{n}, \mathscr{T}_{2}\right) \simeq \mathbb{C}
$$

(тривиальный $G$-модуль).

ДокаЗАТЕЛЬСтво. Все расщепимые супермногообразия, $\overline{0}$-однородные относительно $\mathrm{SL}_{n+1}(\mathbb{C})$, описаны в теореме 4 . Поэтому нам достаточно рассмотреть представления $\varphi$, перечисленные в лемме 1 . Для любого $r \geqslant 0$ последовательность (7) определяет точную последовательность когомологий

$$
\begin{aligned}
0 \longrightarrow H^{0}\left(\mathbb{C P}^{n}, \mathscr{A}_{2 r+1}\right) \stackrel{\alpha^{*}}{\longrightarrow} H^{0}\left(\mathbb{C P}^{n}, \mathscr{T}_{2 r}\right) \stackrel{\beta^{*}}{\longrightarrow} H^{0}\left(\mathbb{C P}^{n}, \mathscr{B}_{2 r}\right) \\
\stackrel{\delta^{*}}{\longrightarrow} H^{1}\left(\mathbb{C P}^{n}, \mathscr{A}_{2 r+1}\right) \stackrel{\alpha^{*}}{\longrightarrow} H^{1}\left(\mathbb{C P}^{n}, \mathscr{T}_{2 r}\right) \stackrel{\beta^{*}}{\longrightarrow} H^{1}\left(\mathbb{C P}^{n}, \mathscr{B}_{2 r}\right) .
\end{aligned}
$$

При этом гомоморфизмы $\alpha^{*}$ и $\beta^{*}$ эквивариантны, так что из $H^{1}\left(\mathbb{C P}^{n}, \mathscr{T}_{2 r}\right)^{G} \neq 0$ следует, что $H^{1}\left(\mathbb{C P}^{n}, \mathscr{A}_{2 r+1}\right)^{G} \neq 0$ или $H^{1}\left(\mathbb{C P}^{n}, \mathscr{B}_{2 r}\right)^{G} \neq 0$.

В предложении 4 описаны все случаи, когда 1 -когомологии пучков $\mathscr{A}_{r}$ и $\mathscr{B}_{r}$ отличны от 0. Рассматривая соответствуюшие представления $\Phi_{1}$, мы видим, что во всех случаях из $H^{1}\left(\mathbb{C P}^{n}, \mathscr{A}_{2 r+1}\right)^{\mathrm{Sp}_{2 r}(\mathbb{C})} \neq 0$ следует, что

$$
H^{1}\left(\mathbb{C P}^{n}, \mathscr{A}_{2 r+1}\right)^{\mathrm{Sp}_{2 r}(\mathbb{C})}=H^{1}\left(\mathbb{C P}^{n}, \mathscr{A}_{2 r+1}\right)^{\mathrm{SL}_{n+1}(\mathbb{C})}=H^{1}\left(\mathbb{C P}^{n}, \mathscr{A}_{2 r+1}\right),
$$

и аналогично, из $H^{1}\left(\mathbb{C P}^{n}, \mathscr{B}_{2 r}\right)^{\mathrm{Sp}_{2 r}(\mathbb{C})} \neq 0$ следует, что

$$
H^{1}\left(\mathbb{C P}^{n}, \mathscr{B}_{2 r}\right)^{\mathrm{Sp}_{2 r}(\mathbb{C})}=H^{1}\left(\mathbb{C P}^{n}, \mathscr{B}_{2 r}\right)^{\mathrm{SL}_{n+1}(\mathbb{C})}=H^{1}\left(\mathbb{C P}^{n}, \mathscr{B}_{2 r}\right),
$$

причем инвариантные когомологии нетривиальны только в следуюших случаях:

1) $n=m \geqslant 2, \varphi=\rho^{*} d^{-1}, H^{1}\left(\mathbb{C P}^{n}, \mathscr{B}_{2}\right) \simeq \mathbb{C}$;

2) $n=m=3, \varphi=\rho d^{-1}, H^{1}\left(\mathbb{C P}^{3}, \mathscr{A}_{3}\right) \simeq \mathbb{C}$;

3) $n=m=2, \varphi=d^{k}+d^{l}$, где $k+l=-3, H^{1}\left(\mathbb{C P}^{2}, \mathscr{B}_{2}\right) \simeq \mathbb{C}$. 
В случае 1) в силу (34) имеем $\varphi=\tau^{*}$, откуда $\mathbf{E}=\mathbf{T}(M)^{*}$ и $\bigwedge \mathscr{E} \simeq \Omega$. Кроме того, $\mathscr{A}_{r}=\mathscr{B}_{r}$. Используя (11), получаем

$$
H^{1}\left(\mathbb{C P}^{n}, \mathscr{T}_{2}\right) \simeq H^{1}\left(\mathbb{C P}^{n}, \mathscr{B}_{3}\right) \oplus H^{1}\left(\mathbb{C P}^{n}, \mathscr{B}_{2}\right) \simeq \mathbb{C}
$$

в силу предложения 4 .

В случае 2) воспользуемся точной последовательностью (36), где $n=3, r=1$. Из предложения 4 видим, что

$$
H^{1}\left(\mathbb{C P}^{3}, \mathscr{A}_{3}\right)=\mathbb{C}, \quad H^{1}\left(\mathbb{C P}^{3}, \mathscr{B}_{2}\right)=0 .
$$

С другой стороны, $H^{0}\left(\mathbb{C P}^{3}, \mathscr{B}_{2}\right) \simeq \mathbb{C}$. Действительно, в силу предложения 3 представление $\mathrm{B}_{2}$ разлагается в нашем случае на неприводимые компоненты со старшими весами $\lambda_{1}-\lambda_{3}$ и 0 , из которых второй вес доминантен. Наконец, заметим, что $H^{0}\left(\mathbb{C P}^{3}, \mathscr{T}_{2}\right)=0$. Это следует из результатов работы [13, п. 2.2.1] (см. также [14]), где вычислены супералгебры Ли векторных полей на расщепимых однородных супермногообразиях, соответствуюших нетривиальным неприводимым представлениям $\varphi$. В результате из (36) вытекает последовательность

$$
0 \longrightarrow \mathbb{C} \longrightarrow \mathbb{C} \longrightarrow H^{1}\left(\mathbb{C P}^{3}, \mathscr{T}_{2}\right) \longrightarrow 0
$$

Из ее точности, очевидно, следует, что $H^{1}\left(\mathbb{C P}^{3}, \mathscr{T}_{2}\right)=0$.

В случае 3$) \mathscr{A}_{3}=0$, откуда $\mathscr{T}=\mathscr{B}_{2}$. Поэтому $H^{1}\left(\mathbb{C P}^{2}, \mathscr{T}_{2}\right) \simeq \mathbb{C}$. Таким образом, теорема доказана.

ЗАмЕчАнИЕ. Из предложения 4 и теоремы 5 легко следует, что если супермногообразие $\left(\mathbb{C P}^{n}, \wedge \mathscr{E}_{\varphi}\right)$, где $\varphi$ - представление типа (I) или (II), не является однородным, то $H^{1}\left(\mathbb{C P}^{n}, \mathscr{T}_{p}\right)=0$ для всех $p \geqslant 0$.

В заключение мы дадим полное описание когомологий касательного пучка для некоторых расщепимых супермногообразий.

Как было отмечено при доказательстве теоремы 8 , в случае, когда $\varphi \simeq \rho^{*} d^{-1}$, структурный пучок $\bigwedge \mathscr{E}$ изоморфен пучку голоморфных форм $\Omega$, откуда в силу (11) $\mathscr{T}_{r} \simeq \mathscr{A}_{r+1} \oplus \mathscr{A}_{r}$. Когомологии пучков $\mathscr{A}_{r}=\Theta \otimes \Omega^{r}$ нетрудно выгислить при помощи теоремы Ботта, используя предложение 3 . Поскольку эти вычисления уже были опубликованы в [15], мы приведем их результаты без доказательства.

ТЕОРема 9. Пусть $\mathscr{A}_{r}=\Theta \otimes \Omega^{r}$ и $\mathscr{T}$ - касательный пучок супермногообразия $\left(\mathbb{C P}^{n}, \Omega\right)$. Тогда

$$
\begin{aligned}
H^{0}\left(\mathbb{C P}^{n}, \mathscr{A}_{0}\right) & \simeq \mathfrak{s l}_{n+1}(\mathbb{C}) \\
H^{r-1}\left(\mathbb{C P}^{n}, \mathscr{A}_{r}\right) & \simeq \mathbb{C}, 1 \leqslant r \leqslant n, \\
H^{q}\left(\mathbb{C P}^{n}, \mathscr{A}_{r}\right) & =0 \text { в остальньх случаях }
\end{aligned}
$$

Если $\theta_{r}-$ базисный әлемент пространства $H^{r-1}\left(\mathbb{C P}^{n}, \mathscr{A}_{r}\right)$, то

$$
\begin{aligned}
H^{0}\left(\mathbb{C P}^{n}, \mathscr{T}_{-1}\right) & \simeq \mathfrak{s l}_{n+1}(\mathbb{C}), \\
H^{0}\left(\mathbb{C P}^{n}, \mathscr{T}_{0}\right) & \simeq \mathfrak{s l}_{n+1}(\mathbb{C}) \oplus \mathbb{C}(\text { как алгебрь Ли }), \\
H^{r-1}\left(\mathbb{C P}^{n}, \mathscr{T}_{r}\right) & =\left\langle l^{*} \theta_{r}\right\rangle \text { при } 1 \leqslant r \leqslant n, \\
H^{r}\left(\mathbb{C P}^{n}, \mathscr{T}_{r}\right) & =\left\langle\alpha^{*} \theta_{r+1}\right\rangle \text { при } 1 \leqslant r \leqslant n-1, \\
H^{q}\left(\mathbb{C P}^{n}, \mathscr{T}_{r}\right) & =0 \text { в остальных случаях. }
\end{aligned}
$$

Доказательство следующей теоремы легко выводится из теоремы Ботта и точной последовательности когомологий, связанной с (7). 
ТЕОРема 10. Пусть $\left(\mathbb{C P}^{2}, \mathscr{O}\right)$ - расщепимое однородное супермногообразие размерности $2 \mid 2$, структурный пучок которого определен представлением $\varphi=d^{-1}+d^{-2}$. Тогда в ненулевых группах когомологий $H^{q}\left(\mathbb{C P}^{2}, \mathscr{T}_{p}\right)$ индуцируются следующие представления группы $\mathrm{SL}_{3}(\mathbb{C})$ :

$$
\begin{aligned}
& \text { в } H^{0}\left(\mathbb{C P}^{2}, \mathscr{T}_{-1}\right): R\left(-\lambda_{3}\right)+R\left(-2 \lambda_{3}\right), \\
& \text { в } H^{0}\left(\mathbb{C P}^{2}, \mathscr{T}_{0}\right): \operatorname{Ad}+R\left(-\lambda_{3}\right)+2 R(0), \\
& \text { в } H^{0}\left(\mathbb{C P}^{2}, \mathscr{T}_{1}\right): R\left(\lambda_{1}\right), \\
& \text { в } H^{1}\left(\mathbb{C P}^{2}, \mathscr{T}_{2}\right): R(0) .
\end{aligned}
$$

Остальные группы когомологий тривиальны.

\section{Список литературы}

1. Манин Ю. И. Калибровочные поля и комплексная геометрия. М.: Наука, 1984.

2. Bunegina V.A., Onishchik A.L. Homogeneous supermanifolds associated with the complex projective line // J. Math. Sciences. 1996. V. 82. № 4. P. 3503-3527.

3. Bunegina V. A., Onishchik A. L. Two families of flag supermanifolds // Differential Geom. Appl. 1994. V. 4. № 4. P. 329-360.

4. Платонова О. В. Об однородньх супермногообразиях размерности 2 | 2 // УМН. 1995. T. 50. №6. C. 205-206.

5. Платонова О.В. Об однородных супермногообразиях, связанных с комплексньп проективньм пространством // Акт. проблемы естеств. наук. Математика. Информатика. Ярославль: Яросл. ун-т, 1995. С. 46-47.

6. Онищик A. Л. Транзитивные супералгебры Ли векторных полей // Деп. в ВИНИТИ 12.06.86, № 4329-B.

7. Kolár I., Michor P. W., Slovák J. Natural Operations in Differential Geometry. Berlin: Springer-Verlag, 1993.

8. Akhiezer D. N. Lie group actions in complex analysis. Braunschweig: Vieweg \& Sohn, 1995.

9. Онищик A. Л. Топология транзитивных групп преобразований. М.: Физматлит, 1995.

10. Snow D. M. Spanning homogeneous vector bundles // Comment. Math. Helv. 1989. V. 64. № 3. P. 395-400.

11. Винберг Э. Б., Онищик A. Л. Семинар по группам Ли и алгебраическим группам.. М.: Наука, 1988.

12. Bott R. Homogeneous vector bundles // Ann. of Math. (2). 1957. V. 66. P. 203-248.

13. Серов A. A. Супералгебры Ли векторных полей на комплексных фолаговых супермногообразиях // Деп. в ВИНИТИ 26.01.87, №610-В.

14. Онищик А.Л., Серов А. А. Супералгебры Ли векторных полей на расщепимых фолаговых супермногообразиях // Докл. АН СССР. 1988. Т. 300. С. 284-287.

15. Onishchik A.L. About derivations and vector-valued differential forms // Inst. Math. Ruhr-Universität Bochum. Bericht № 181, 1995. 